TRANSACTIONS OF THE

AMERICAN MATHEMATICAL SOCIETY

Volume 357, Number 4, Pages 1497-1524

S 0002-9947(04)03734-1

Article electronically published on November 23, 2004

\title{
MEASURABLE KAC COHOMOLOGY FOR BICROSSED PRODUCTS
}

\author{
SAAD BAAJ, GEORGES SKANDALIS, AND STEFAAN VAES
}

\begin{abstract}
We study the Kac cohomology for matched pairs of locally compact groups. This cohomology theory arises from the extension theory of locally compact quantum groups. We prove a measurable version of the Kac exact sequence and provide methods to compute the cohomology. We give explicit calculations in several examples using results of Moore and Wigner.
\end{abstract}

\section{INTRODUCTION}

In order to construct, in a systematic way, examples of finite quantum groups, G. Kac developed in [13] a method to obtain non-trivial (i.e. non-commutative and non-cocommutative) quantum groups as extensions of a finite group by a finite group dual. Such an extension of a finite group $G_{1}$ with the dual of a finite group $G_{2}$ is described by the following data:

- a large group $G$ such that $G_{1}$ and $G_{2}$ are subgroups of $G$ satisfying $G_{1} \cap G_{2}=$ $\{e\}$ and $G=G_{1} G_{2}$ (we say that $G_{1}, G_{2} \subset G$ is a matched pair),

- a compatible pair of 2-cocycles (see (5) below).

Two extensions are isomorphic if and only if the matched pairs are the same and the pairs of 2-cocycles are cohomologous. As such, there appears a natural cohomology group associated with a matched pair. G. Kac found in 13 an exact sequence which permits to calculate this cohomology group in terms of the usual cohomology groups of $G_{1}, G_{2}$ and $G$ with coefficients in $\mathbb{T}$, the group of complex numbers of modulus 1.

The above theory of extensions has been generalized to the framework of locally compact quantum groups (in the sense of Kustermans and the third author [14, 15]) by Vainerman and the third author [23]: extensions in the category of locally compact quantum groups are exactly described using matched pairs with cocycles. Again, there appears a natural cohomology group. The aim of this paper is to study this cohomology group, to prove a version of the Kac exact sequence in a locally compact setting and to compute the cohomology in concrete examples. As such, we shall provide a precise explanation for the calculations in 24].

Given a matched pair of locally compact groups (see Definition 2.1), the first two authors introduced in [3] an alternative notion of cocycles and, hence, another cohomology group. An awkward, but straightforward, calculation yields that both

Received by the editors October 24, 2003.

2000 Mathematics Subject Classification. Primary 22D05; Secondary 55N99, 20 J06.

Key words and phrases. Measurable cohomology, locally compact quantum groups, extensions, Kac exact sequence.

(C)2004 American Mathematical Society Reverts to public domain 28 years from publication 
cohomology groups agree for matched pairs of discrete (and in particular, finite) groups. Below, we will use a more elegant approach that permits us to conclude that both cohomologies agree for the most general matched pairs of locally compact groups.

After the fundamental work of Kac 13, matched pairs of locally compact groups have been studied by Majid [18, 19] in order to construct examples of Kac algebras. His definition of a matched pair of locally compact groups $G_{1}, G_{2} \subset G$ requires that $G_{1} \cap G_{2}=\{e\}$ and that the multiplication map $G_{1} \times G_{2} \rightarrow G$ is a homeomorphism onto $G$. The first two authors [3, 4] gave a more general definition, allowing $G_{1} G_{2}$ to be an open subset of $G$ with complement of measure zero. Using such a matched pair, they constructed a multiplicative unitary, given by a pentagonal transformation. The most general definition of a matched pair has been introduced in 23] by Vainerman and the third author and in [5] by the authors, requiring only that $G_{1} \cap G_{2}=\{e\}$ and that $G_{1} G_{2}$ has a complement of measure zero. We remark here that examples of such matched pairs, with $G_{1} G_{2}$ having empty interior, were given in [5] and used to construct examples of locally compact quantum groups with remarkable topological properties.

We mention that algebraic work on matched pairs and Kac cohomology for Hopf algebras and Lie algebras has been done in e.g. 1, 20, 22.

\section{Preliminaries}

In this paper, all locally compact spaces will be supposed second countable. We denote by $\mathbb{T}$ the group of complex numbers of modulus 1 , which we will often write additively through the identification with $\mathbb{R} / \mathbb{Z}$.

Definition 2.1. We call $G_{1}, G_{2} \subset G$ a matched pair of locally compact groups if $G_{1}, G_{2}$ are closed subgroups of the locally compact group $G$ such that $G_{1} \cap G_{2}=\{e\}$ and $G \backslash G_{1} G_{2}$ has Haar measure zero.

Notation 2.2. Given a matched pair $G_{1}, G_{2} \subset G$ of locally compact groups, we define, almost everywhere on $G$,

$$
\begin{gathered}
p_{1}: G \rightarrow G_{1} \quad, \quad p_{2}: G \rightarrow G_{2} \quad \text { such that } \quad x=p_{1}(x) p_{2}(x), \\
q_{1}: G \rightarrow G_{1} \quad, \quad q_{2}: G \rightarrow G_{2} \quad \text { such that } \quad x=q_{2}(x) q_{1}(x) .
\end{gathered}
$$

We will deal with cohomology theories with coefficients in Polish $G$-modules. Therefore, the following will be useful to us.

Notation 2.3. Let $X$ be a standard Borel space equipped with a Borel measure class. Let $A$ be a Polish space. We define $\mathrm{L}(X, A)$ to be the set of equivalence classes of Borel measurable functions of $X$ to $A$ identifying functions equal almost everywhere. Choosing a finite measure $\mu$ on $X$ in the given measure class and a bounded complete metric $\rho$ on $A$, we can define

$$
\rho_{\mu}(F, G):=\int \rho(F(x), G(x)) d \mu(x) \text { for all } F, G \in \mathrm{L}(X, A) .
$$

In this way, $\mathrm{L}(X, A)$ is a Polish space. The topology on $\mathrm{L}(X, A)$ does not depend on the choice of $\mu$ or $\rho$; see the Corollary to Proposition 6 in [21].

In Theorem 1 of [21], the Fubini theorem is used to prove that there are natural isomorphisms $\mathrm{L}(X, \mathrm{~L}(Y, A)) \cong \mathrm{L}(X \times Y, A) \cong \mathrm{L}(Y, \mathrm{~L}(X, A))$ for all Polish spaces $A$. 
We finally introduce the measurable cohomology of a locally compact group $G$, as studied by Moore [21] and D. Wigner [25].

Let $G$ be a locally compact group and let $A$ be a Polish $G$-module. We write face operators

$$
\partial_{i}: G^{n+1} \rightarrow G^{n}: \partial_{i}\left(g_{0}, \ldots, g_{n}\right)=\left\{\begin{array}{l}
\left(g_{1}, \ldots, g_{n}\right) \text { if } i=0, \\
\left(g_{0}, \ldots, g_{i-1} g_{i}, \ldots, g_{n}\right) \text { if } i=1, \ldots, n, \\
\left(g_{0}, \ldots, g_{n-1}\right) \text { if } i=n+1
\end{array}\right.
$$

Dualizing, we can write at least two natural measurable cochain complexes. First, consider

(1) $\mathrm{d}_{i}: \mathrm{L}\left(G^{n}, A\right) \rightarrow \mathrm{L}\left(G^{n+1}, A\right):\left(\mathrm{d}_{i} F\right)(\vec{g})=\left\{\begin{array}{l}g_{0} \cdot F\left(\partial_{0} \vec{g}\right) \text { if } i=0, \\ F\left(\partial_{i} \vec{g}\right) \text { if } i=1, \ldots, n+1,\end{array}\right.$

where $\vec{g}=\left(g_{0}, \ldots, g_{n}\right)$ and

(2) $\mathrm{d}: \mathrm{L}\left(G^{n}, A\right) \rightarrow \mathrm{L}\left(G^{n+1}, A\right): \mathrm{d}=\sum_{i=0}^{n+1}(-1)^{i} \mathrm{~d}_{i}$.

Definition 2.4. The cohomology of the cochain complex $\left(\mathrm{L}\left(G^{n}, A\right)\right)_{n}$ defined above is denoted by $H(G, A)$ and called the measurable cohomology of the locally compact group $G$ with coefficients in the Polish $G$-module $A$.

Instead of using $\mathrm{L}\left(G^{n}, A\right)$, we can use the $\mathbb{Z}$-module $\mathcal{F}_{\text {Borel }}\left(G^{n}, A\right)$ of Borel measurable functions from $G^{n}$ to $A$. We define the coboundary with the same formula as in (11)-(2). Moore 21] proved that the obvious cochain transformation $\left(\mathcal{F}_{\text {Borel }}\left(G^{n}, A\right)\right) \rightarrow\left(\mathrm{L}\left(G^{n}, A\right)\right)$ is a cohomology isomorphism.

In fact, there is more. Let $G$ be a locally compact group and consider a certain category of $G$-modules. Suppose that $H(G, A)$ and $\tilde{H}(G, A)$ are two cohomology theories satisfying the Buchsbaum criterion $[9]$ :

- every short exact sequence of $G$-modules gives rise, in a natural way, to a long exact cohomology sequence,

- effaceability, i.e. for every $\alpha \in H^{n}(G, A)$ there exists a short exact sequence $0 \rightarrow A \rightarrow B \rightarrow C \rightarrow 0$ such that $\alpha$ is 0 in $H^{n}(G, B)$.

Now if $H^{0}(G, A)$ and $\tilde{H}^{0}(G, A)$ are naturally isomorphic, we can conclude that $H^{n}(G, A)$ and $\tilde{H}^{n}(G, A)$ are naturally isomorphic for all $n$. Further, any natural sequence of homomorphisms $H^{n}(G, A) \rightarrow \tilde{H}^{n}(G, A)$ which is connected (i.e. respects the long exact cohomology sequences) and which gives an isomorphism for $n=0$, will be an isomorphism for all $n$.

Another way of describing the measurable cohomology of $G$ is through the right notion of a free resolution.

Definition 2.5. We say that a Polish $G$-module $A$ is free if there exists a Polish $G$-module $B$ such that $A \cong \mathrm{L}(G, B)$.

Let $A$ be a Polish $G$-module. We call

$$
0 \longrightarrow A \longrightarrow A_{0} \longrightarrow A_{1} \longrightarrow \cdots
$$

a resolution of $A$, if all $A_{i}$ are Polish $G$-modules, the arrows are $G$-equivariant and continuous, and the sequence in the previous equation is exact. 
We say that the resolution in (3) is a free resolution of $A$ if every Polish $G$-module $A_{i}, i \geq 0$, is free.

Whenever $0 \longrightarrow A \longrightarrow A_{0} \longrightarrow A_{1} \longrightarrow \cdots$ is a free resolution of the Polish $G$ module $A$, the measurable cohomology $H(G, A)$ is the cohomology of the complex

$$
A_{0}^{G} \longrightarrow A_{1}^{G} \longrightarrow \cdots,
$$

where $A_{i}^{G}$ denotes the $G$-fixed points of $A_{i}$. Again, as above, if we have two free resolutions of $A$ :

$$
0 \longrightarrow A \longrightarrow A_{0} \longrightarrow A_{1} \longrightarrow \cdots \quad \text { and } \quad 0 \longrightarrow A \longrightarrow B_{0} \longrightarrow B_{1} \longrightarrow \cdots
$$

and if we are given continuous $G$-equivariant homomorphisms $A_{i} \rightarrow B_{i}$ intertwining the two free resolutions, then these homomorphisms induce a cohomology isomorphism.

In Section 7 we explain the methods developed by D. Wigner to compute the measurable cohomology $H(G, A)$ in certain cases.

\section{Two KaC 2-COHOMOlogy GRoups And the KaC BICOMPleX}

The Kac 2-cohomology appears in two natural ways. In both pictures, it is at first somehow awkward to write the cocycle relations. We will see below how a much more natural way of writing these relations can be obtained. This will also allow us to unify both pictures and prove that they define the same 2-cohomology group.

3.1. 2-cohomology of matched pairs. First of all, we want to classify extensions

$$
e \longrightarrow\left(\mathrm{L}^{\infty}\left(G_{1}\right), \Delta_{1}\right) \longrightarrow(M, \Delta) \longrightarrow\left(\mathcal{L}\left(G_{2}\right), \hat{\Delta}_{2}\right) \longrightarrow e,
$$

where $(M, \Delta)$ is a locally compact quantum group. Here, we do not explain the notion of such an extension in the framework of locally compact quantum groups (see Definition 3.2 in [23]). All good (i.e., cleft) extensions can be written as a cocycle bicrossed product of $G_{1}$ and $G_{2}$; see Theorem 3.6 in 23. Using Remark 5.3 in [5] and Lemma 4.11 in [23], this means that

- there exists a locally compact group $G$ such that $G_{1}, G_{2}$ are closed subgroups of $G$ forming a matched pair in the sense of Definition 2.1

- there exists a compatible pair $(\mathcal{U}, \mathcal{V})$ of 2-cocycles on the matched pair $G_{1}, G_{2}$. This means that $\mathcal{U}: G_{2} \times G_{1} \times G_{1} \rightarrow \mathbb{T}$ and $\mathcal{V}: G_{2} \times G_{2} \times G_{1} \rightarrow \mathbb{T}$ are measurable maps satisfying the (awkward) relations

$$
\begin{gathered}
\mathcal{U}\left(p_{2}(s g), h, k\right) \overline{\mathcal{U}}(s, g h, k) \mathcal{U}(s, g, h k) \overline{\mathcal{U}}(s, g, h)=1, \\
\mathcal{V}(t, r, g) \overline{\mathcal{V}}(s t, r, g) \mathcal{V}(s, t r, g) \overline{\mathcal{V}}\left(s, t, p_{1}(r g)\right)=1, \\
\mathcal{U}(t, g, h) \overline{\mathcal{U}}(s t, g, h) \mathcal{U}\left(s, p_{1}(t g), p_{1}\left(p_{2}(t g) h\right)\right) \mathcal{V}\left(p_{2}\left(s p_{1}(t g)\right), p_{2}(t g), h\right) \times \\
\overline{\mathcal{V}}(s, t, g h) \mathcal{V}(s, t, g)=1,
\end{gathered}
$$

for almost all $s, t, r \in G_{2}$ and $g, h, k \in G_{1}$.

The locally compact quantum group $(M, \Delta)$ is the cocycle bicrossed product constructed with this data of a matched pair and a compatible pair of 2-cocycles.

From Proposition 3.8 in [23], we know that two extensions are isomorphic if and only if the associated matched pairs of locally compact groups are the same and 
there exists a measurable map $\mathcal{R}: G_{2} \times G_{1} \rightarrow \mathbb{T}$ such that the pairs of 2-cocycles $(\mathcal{U}, \mathcal{V})$ differ by a trivial pair of 2-cocycles $\left(\mathcal{U}_{\mathcal{R}}, \mathcal{V}_{\mathcal{R}}\right)$ defined by

$$
\begin{aligned}
\mathcal{U}_{\mathcal{R}}(s, g, h) & =\overline{\mathcal{R}}\left(p_{2}(s g), h\right) \mathcal{R}(s, g h) \overline{\mathcal{R}}(s, g), \\
\mathcal{V}_{\mathcal{R}}(s, t, g) & =\mathcal{R}(t, g) \overline{\mathcal{R}}(s t, g) \mathcal{R}\left(s, p_{1}(t g)\right) .
\end{aligned}
$$

This leads us to the following definition (Terminology 4.21 in [23]).

Definition 3.1. Let $G_{1}, G_{2} \subset G$ be a matched pair of locally compact groups. The associated group of extensions is defined as the group of pairs of 2-cocycles $(\mathcal{U}, \mathcal{V}) \in \mathrm{L}\left(G_{2} \times G_{1}^{2}, \mathbb{T}\right) \oplus \mathrm{L}\left(G_{2}^{2} \times G_{1}, \mathbb{T}\right)$ satisfying (55), modulo the subgroup of trivial cocycles defined by (6).

As a conclusion, we see that extensions (44) are classified by a matched pair $G_{1}, G_{2} \subset G$ and the associated group of extensions.

3.2. 2-cohomology of pentagonal transformations. Secondly, fix a matched pair $G_{1}, G_{2} \subset G$. There is an associated bicrossed product locally compact quantum group $(M, \Delta)$ with multiplicative unitary $W$. By definition (see [3]), a multiplicative unitary is a unitary operator on a tensor square $H \otimes H$ of a Hilbert space $H$, satisfying the pentagonal equation

$$
W_{12} W_{13} W_{23}=W_{23} W_{12}
$$

on $H \otimes H \otimes H$. For the bicrossed product of $G_{1}, G_{2} \subset G$, this multiplicative unitary is given, as in [3, 4] and up to some identifications, by the formula $(W \xi)(x, y)=$ $d(x, y)^{1 / 2} \xi(w(x, y))$ for $x, y \in G$, where

$$
w(x, y)=\left(x p_{1}\left(p_{2}(x)^{-1} y\right), p_{2}(x)^{-1} y\right)
$$

and where $d(x, y)$ is the Radon-Nikodym derivative making $W$ a unitary operator on $L^{2}(G \times G)$. In Définition 8.24 of [3], a 2-cocycle for the matched pair $G_{1}, G_{2} \subset G$ is defined as a measurable function $\tilde{\theta}: G \times G \rightarrow \mathbb{T}$ such that $W_{\tilde{\theta}}:=\tilde{\theta} W$ is still a multiplicative unitary. Here, $\tilde{\theta}$ denotes as well the multiplication operator by the function $\tilde{\theta}$. A trivial 2-cocycle is a 2-cocycle of the form $\tilde{\theta}(x, y)=t(x, y) \overline{t(w(x, y))}$, where $t(x, y)=a(x) a(y)$ for some measurable function $a: G \rightarrow \mathbb{T}$. In that case $W_{\tilde{\theta}}=(a \otimes a) W\left(a^{*} \otimes a^{*}\right)$, which motivates why such a $\tilde{\theta}$ is considered to be trivial. Dividing the group of 2-cocycles by trivial 2-cocycles, we get again a 2-cohomology group. For finite groups $G_{1}, G_{2}$, the computational argument in Section 4.4 of 23. allows us to conclude that this 2-cohomology group is isomorphic with the 2cohomology group defined in Definition 3.1 using pairs $(\mathcal{U}, \mathcal{V})$. The more delicate general case will be dealt with below.

Observing that $w=w_{1} \circ w_{2}$, where $w_{1}(x, y)=\left(x p_{1}(y), y\right)$ and $w_{2}(x, y)=$ $\left(x, p_{2}(x)^{-1} y\right)$, we write $W=W_{2} W_{1}$. To simplify formulas, we may as well define a 2-cocycle as a measurable function $\theta: G \times G \rightarrow \mathbb{T}$ such that $W_{2} \theta W_{1}$ is a multiplicative unitary. Of course, one can pass from $\theta$ to $\tilde{\theta}$ by the formula $\tilde{\theta}(x, y)=\theta\left(w_{2}(x, y)\right)$. The 2-cocycle relation for $\theta$ becomes

$$
\theta(x, y) \theta\left(x p_{1}(y), p_{2}(y) z\right) \theta(y, z)=\theta\left(p_{2}(x) y, z\right) \theta\left(x, y p_{1}(z)\right)
$$

for almost all $x, y, z \in G$. Trivial 2-cocycles are given by the formula

$$
\theta(x, y)=a(x) a\left(p_{2}(x) y\right) \overline{a\left(x p_{1}(y)\right)} \overline{a(y)}
$$

for some measurable function $a: G \rightarrow \mathbb{T}$ and almost all $x, y \in G$. 
Definition 3.2. Let $G_{1}, G_{2} \subset G$ be a matched pair. The 2-cohomology group associated with the pentagonal transformation (7) is defined as the group of cocycles $\theta \in \mathrm{L}(G \times G, \mathbb{T})$ satisfying $(8)$, divided by the subgroup of trivial cocycles defined by (9).

3.3. The Kac bicomplex. Now fix a matched pair $G_{1}, G_{2} \subset G$. Define the closed subspace $\Gamma_{11} \subset G_{1} \times G_{1} \times G_{2} \times G_{2}$ as follows:

$$
\Gamma_{11}:=\left\{h \prod_{t}^{s} g \mid g, h \in G_{1}, s, t \in G_{2}, s g=h t\right\} .
$$

Lemma 3.3. The maps

$$
\Gamma_{11} \rightarrow G_{1} \times G_{2}: h \prod_{t}^{s} g \mapsto\left\{\begin{array}{l}
(g, s), \\
(g, t), \\
(h, s), \\
(h, t)
\end{array} \quad \text { and } \quad \Gamma_{11} \rightarrow G: h \prod_{t}^{s} g \mapsto s g\right.
$$

are injective. Their ranges have complement of measure zero and all these maps define the same measure class on $\Gamma_{11}$.

Proof. This follows immediately from Proposition 3.2 in 5 and the remarks following that proposition.

When $x \in G_{1} G_{2} \cap G_{2} G_{1}$ (and, as follows from the previous lemma, almost all $x \in G$ are like that), we sometimes write $h \stackrel{s}{x}_{t}^{t} g$ to denote the element $h \bigsqcup_{t}^{s} g \in$

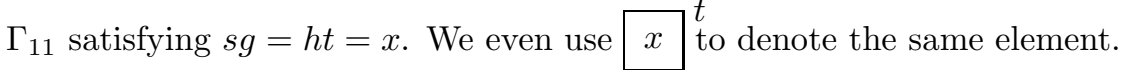

So, we defined the space $\Gamma_{11}$ by labelling the edges of a square. We will use this to give a non-equivariant image of our cohomology theory. There is an analogous equivariant image. We define $\Upsilon_{11} \subset G^{4}$ as follows:

$$
\Upsilon_{11}:=\left\{\square_{z}^{x} \mid x, y, z, w \in G, x^{-1} z, y^{-1} w \in G_{1}, \quad x^{-1} y, z^{-1} w \in G_{2}\right\} .
$$

There is a natural action of $G$ on $\Upsilon_{11}$ given by

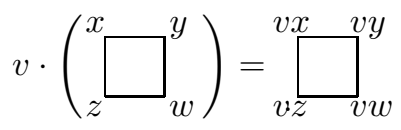

and a natural homeomorphism $G \times \Gamma_{11} \rightarrow \Upsilon_{11}$ given by

$$
\left(v, \quad h \frac{s}{t} g\right) \mapsto \bigsqcup_{v h}^{v} g x
$$


We define more generally the space $\Gamma_{p q}$ (which is, stricto sensu, a closed subspace of $\left.G_{1}^{p(q+1)} \times G_{2}^{(p+1) q}\right)$ consisting of elements $X \in \Gamma_{p q}$ defined by

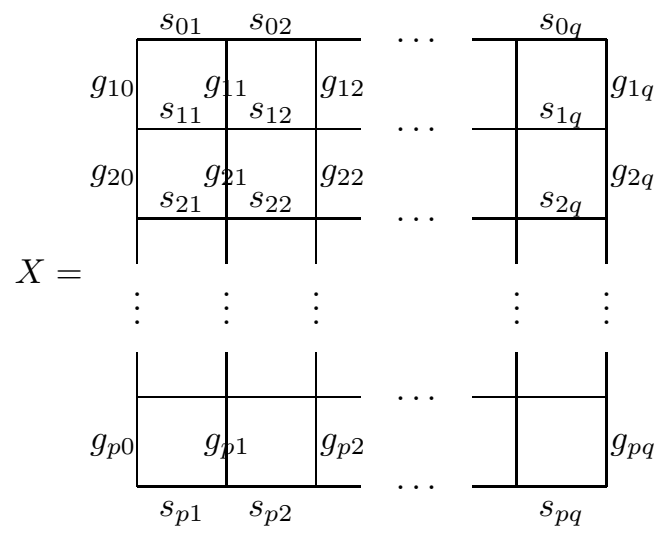

where all $g_{i j} \in G_{1}, s_{i j} \in G_{2}$ and every small square of the above picture belongs to $\Gamma_{11}$. This means, e.g., that $s_{12} g_{22}=g_{21} s_{22}$. More generally, this means that if one chooses two vertices in $X$ and a path between them, then the result of the multiplication of all the edges along the path does not depend on the chosen path. As such, we define $\Gamma_{p q}$ whenever $p+q \neq 0$. We define $\Gamma_{00}$ to be one point and remark that $\Gamma_{p 0}=G_{1}^{p}$ and $\Gamma_{0 q}=G_{2}^{q}$.

Of course, we have again an analogous equivariant image $\Upsilon_{p q} \subset G^{(p+1) \times(q+1)}$, consisting of $(p+1) \times(q+1)$-matrices $Y$ with entries in $G$

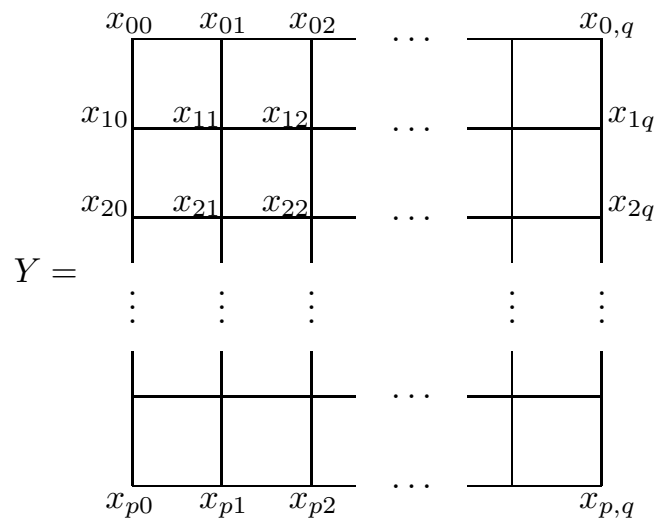

such that the elements on a fixed row define the same element of $G / G_{2}$ and the elements on a fixed column define the same element of $G / G_{1}$. More formally, $x_{i j}^{-1} x_{k j} \in G_{1}$ and $x_{i j}^{-1} x_{i k} \in G_{2}$.

Again, we have an action of $v \in G$ on $Y \in \Upsilon_{p q}$, multiplying all $x_{i j}$ in (11) by $v$ on the left. We get a homeomorphism $\Upsilon_{p q} \rightarrow G \times \Gamma_{p q}$, which sends an element $Y$ to the couple $\left(x_{00}, X\right)$, where $X$ is defined by $g_{i j}=x_{i-1, j}^{-1} x_{i j}$ and $s_{i j}=x_{i, j-1}^{-1} x_{i j}$.

Remark 3.4. An element of $\Gamma_{p q}$ is uniquely determined once we know one row and one column. The mapping $\Gamma_{p q} \rightarrow G_{1}^{p} \times G_{2}^{q}$ picking out one column and one row is injective and its image has a complement of measure zero. It follows from Lemma 3.3 that all these mappings induce the same measure class on $\Gamma_{p q}$. 
Also the mapping $\Gamma_{p q} \rightarrow \Gamma_{k, l} \times \Gamma_{p-k, q-l}$, sending a matrix to its upper left and lower right corner, is injective. Its image has a complement of measure zero and the map is a measure class isomorphism.

We define horizontal and vertical face operators on $\Upsilon_{p q}$ :

$$
\begin{aligned}
& \partial_{i}^{\mathrm{h}}: \Upsilon_{p q} \rightarrow \Upsilon_{p, q-1}: \partial_{i}^{\mathrm{h}} \text { removes the } i \text {-th column } \quad(\text { for } i=0, \ldots, q) \text { and } \\
& \partial_{j}^{\mathrm{v}}: \Upsilon_{p q} \rightarrow \Upsilon_{p-1, q}: \partial_{j}^{\mathrm{v}} \text { removes the } j \text {-th row } \quad(\text { for } j=0, \ldots, p) .
\end{aligned}
$$

These face operators are obviously $G$-equivariant, and so we get face operators on $\Gamma_{p q}$, defined as follows. The face $\partial_{i}^{\mathrm{h}}$ contracts the $i$-th column multiplying the adjacent horizontal edges. In a concrete example, this means that

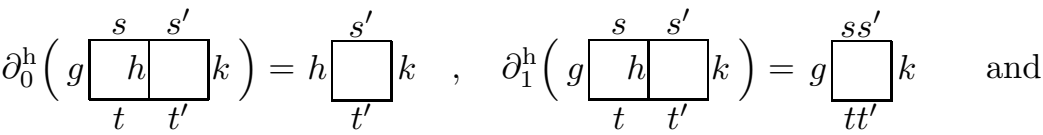

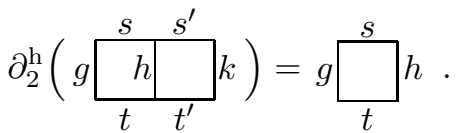

It will be clear how to define the vertical face $\partial_{j}^{v}$ contracting the $j$-th row and multiplying the adjacent vertical edges. Observe that $\partial_{i}^{\mathrm{h}} \partial_{j}^{\mathrm{h}}=\partial_{j-1}^{\mathrm{h}} \partial_{i}^{\mathrm{h}}$ if $i<j$.

Let $A$ be a Polish $G$-module.

Notation 3.5. Following Notation 2.3 , we consider the Polish spaces $\mathrm{L}\left(\Gamma_{p q}, A\right)$ and $\mathrm{L}\left(\Upsilon_{p q}, A\right)$. Using the action of $G$ on $\Upsilon_{p q}$, we turn $\mathrm{L}\left(\Upsilon_{p q}, A\right)$ into a Polish $G$-module, defining

$$
(x \cdot F)(Y)=x \cdot F\left(x^{-1} \cdot Y\right)
$$

for $x \in G$ and $Y \in \Upsilon_{p q}$. We denote by $\mathrm{L}\left(\Upsilon_{p q}, A\right)^{G}$ the $\mathbb{Z}$-module of $G$-invariant elements of $\mathrm{L}\left(\Upsilon_{p q}, A\right)$. We have a natural identification $\mathrm{L}\left(\Gamma_{p q}, A\right) \cong \mathrm{L}\left(\Upsilon_{p q}, A\right)^{G}$.

We define a bicomplex (see [17])

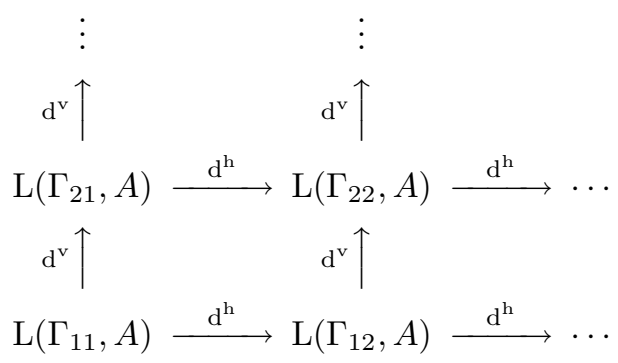

where the arrows can be defined most easily using Notation 3.5 and the equivariant coboundary operators

$$
\begin{aligned}
& \mathrm{d}^{\mathrm{h}}: \mathrm{L}\left(\Upsilon_{p q}, A\right) \rightarrow \mathrm{L}\left(\Upsilon_{p, q+1}, A\right):\left(\mathrm{d}^{\mathrm{h}} F\right)(Y)=\sum_{i=0}^{q+1}(-1)^{i} F\left(\partial_{i}^{\mathrm{h}} Y\right), \\
& \mathrm{d}^{\mathrm{v}}: \mathrm{L}\left(\Upsilon_{p q}, A\right) \rightarrow \mathrm{L}\left(\Upsilon_{p+1, q}, A\right):\left(\mathrm{d}^{\mathrm{v}} F\right)(Y)=\sum_{j=0}^{p+1}(-1)^{j} F\left(\partial_{j}^{\mathrm{v}} Y\right) .
\end{aligned}
$$


A small calculation reveals that, on $\Gamma_{p q}$ rather than $\Upsilon_{p q}$, we get

$$
\begin{aligned}
& \mathrm{d}^{\mathrm{h}}: \mathrm{L}\left(\Gamma_{p q}, A\right) \rightarrow \mathrm{L}\left(\Gamma_{p, q+1}, A\right):\left(\mathrm{d}^{\mathrm{h}} F\right)(X)=s_{01} \cdot F\left(\partial_{0}^{\mathrm{h}} X\right)+\sum_{i=1}^{q+1}(-1)^{i} F\left(\partial_{i}^{\mathrm{h}} X\right), \\
& \mathrm{d}^{\mathrm{v}}: \mathrm{L}\left(\Gamma_{p q}, A\right) \rightarrow \mathrm{L}\left(\Gamma_{p+1, q}, A\right):\left(\mathrm{d}^{\mathrm{v}} F\right)(X)=g_{10} \cdot F\left(\partial_{0}^{\mathrm{v}} X\right)+\sum_{j=1}^{p+1}(-1)^{j} F\left(\partial_{j}^{\mathrm{v}} X\right),
\end{aligned}
$$

when $X \in \Gamma_{p, q+1}$ or $\Gamma_{p+1, q}$ are as in (10). It is clear that we have in fact face operators

$$
\begin{aligned}
& \mathrm{d}_{i}^{\mathrm{h}}: \mathrm{L}\left(\Gamma_{p q}, A\right) \rightarrow \mathrm{L}\left(\Gamma_{p, q+1}\right) \text { for } 0 \leq i \leq q+1 \text { and } \mathrm{d}^{\mathrm{h}}=\sum_{i=0}^{q+1}(-1)^{i} \mathrm{~d}_{i}^{\mathrm{h}}, \\
& \mathrm{d}_{i}^{\mathrm{v}}: \mathrm{L}\left(\Gamma_{p q}, A\right) \rightarrow \mathrm{L}\left(\Gamma_{p+1, q}, A\right) \text { for } 0 \leq i \leq p+1 \text { and } \mathrm{d}^{\mathrm{v}}=\sum_{i=0}^{p+1}(-1)^{i} \mathrm{~d}_{i}^{\mathrm{v}} .
\end{aligned}
$$

For reasons that will become clear later, the elements of $\mathrm{L}\left(\Gamma_{11}, A\right)$ should be considered as 1-cochains rather than 0 -cochains. So, the total complex is defined as

$$
C^{n}(A)=\bigoplus_{p+q=n+1} \mathrm{~L}\left(\Gamma_{p q}, A\right)
$$

and

$$
\mathrm{d}: C^{n}(A) \rightarrow C^{n+1}(A): \mathrm{d}=\mathrm{d}^{\mathrm{h}}+\varepsilon \mathrm{d}^{\mathrm{v}},
$$

where $\varepsilon(F)=(-1)^{q}$ when $F \in \mathrm{L}\left(\Gamma_{p q}, A\right)$.

We shall see in Proposition 4.4 how to define in a natural way $C^{0}=A$ and $\mathrm{d}: C^{0} \rightarrow C^{1}$.

The following proposition is almost obvious.

Proposition 3.6. The group of extensions of the matched pair $G_{1}, G_{2} \subset G$ is precisely the second cohomology group of the total complex defined by (15) with coefficients in the trivial $G$-module $\mathbb{T}$.

Proof. This is just a matter of making the right identifications, taking into account Remark 3.4. If $\mathcal{U}: G_{2} \times G_{1} \times G_{1} \rightarrow \mathbb{T}$ and $\mathcal{V}: G_{2} \times G_{2} \times G_{1} \rightarrow \mathbb{T}$ are measurable maps, we define

$$
\mathcal{U}\left(\square \begin{array}{l}
s \\
h
\end{array}\right)=\mathcal{U}(s, g, h) \text { and } \mathcal{V}\left(\begin{array}{l}
\square^{t} \\
\square
\end{array}\right)=\mathcal{V}(s, t, g) .
$$

Then, $(\mathcal{U}, \mathcal{V}) \in C^{2}(\mathbb{T})$. The equation $\mathrm{d}(\mathcal{U}, \mathcal{V})=0$ agrees precisely with the three equations in (5). Further, if $\mathcal{R}: G_{2} \times G_{1} \rightarrow \mathbb{T}$, we define $\mathcal{R}(\square g)=\mathcal{R}(s, g)$. The equation $\mathrm{d} \mathcal{R}=(\mathcal{U}, \mathcal{V})$ is equivalent with ([6).

Remark 3.7. The locally compact space $\Gamma_{11}$ carries the structure of a double groupoid [7, [8]. The horizontal groupoid $\Gamma_{\mathrm{h}}$ has unit space $\Gamma_{\mathrm{h}}^{(0)}=G_{1}$ which is embedded by 


$$
\begin{aligned}
& g \mapsto g \overbrace{e}^{e} g \text {. The source and range maps are defined by } \\
& \operatorname{Source}\left(h{\underset{t}{s}}_{t}^{s} g\right)=g \text { and } \operatorname{Range}\left(h \prod_{t}^{s} g\right)=h .
\end{aligned}
$$

The composition is defined by

$$
h \prod_{t}^{s} g \cdot g \prod_{t^{\prime}}^{s^{\prime}} h^{\prime}=h \prod_{t t^{\prime}}^{s s^{\prime}} h h^{\prime}
$$

Analogously, the same space $\Gamma_{11}$ carries a second groupoid structure, the vertical groupoid $\Gamma_{\mathrm{v}}$, with unit space $\Gamma_{\mathrm{v}}^{(0)}=G_{2}$. Composition is now defined by vertical composition of squares. As such, $\Gamma_{11}$ becomes a double groupoid: if $x, y, z, v$ are such that $(x, y),(z, v) \in \Gamma_{\mathrm{h}}^{(2)}$ and $(x, z),(y, v) \in \Gamma_{\mathrm{v}}^{(2)}$, then $\left(x \cdot \mathrm{h} y, z \cdot_{\mathrm{h}} v\right) \in \Gamma_{\mathrm{v}}^{(2)}$, $\left(x \cdot{ }_{\mathrm{v}} z, y \cdot{ }_{\mathrm{v}} v\right) \in \Gamma_{\mathrm{h}}^{(2)}$ and

$$
\left(x \cdot{ }_{\mathrm{h}} y\right) \cdot{ }_{\mathrm{v}}\left(z \cdot{ }_{\mathrm{h}} v\right)=\left(x \cdot{ }_{\mathrm{v}} z\right) \cdot \mathrm{h}\left(y \cdot{ }_{\mathrm{v}} v\right) .
$$

This is obvious if one just looks at the square

$$
\begin{array}{|l|l|}
\hline x & y \\
\hline z & v \\
\hline
\end{array}
$$

The bicomplex (12) can now be written down analogously for double groupoids and gives a natural candidate for a double groupoid cohomology.

\section{The Kac eXact SEQuence}

We still have a fixed matched pair $G_{1}, G_{2} \subset G$ of locally compact groups. We consider a Polish $G$-module $A$.

Looking at the bicomplex (12), it is natural to add a row and a column and to write

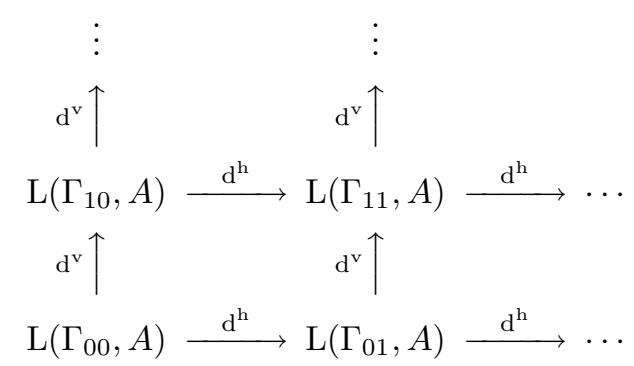

Proposition 4.1. The cohomology of the total bicomplex of (17) is isomorphic with the measurable cohomology of $G$ with coefficients in the Polish G-module A. Moreover, (18) defines an explicit cohomology isomorphism. The inverse isomorphism is given explicitly in Remark 4.2.

Proof. In order to prove that the cohomology of the total bicomplex of (17) is precisely the measurable cohomology of $G$, we consider the $G$-equivariant bicomplex $\left(\mathrm{L}\left(\Upsilon_{p q}, A\right)\right)_{p, q \geq 0}$ and embed $A \hookrightarrow \mathrm{L}\left(\Upsilon_{00}, A\right)$ as constant functions. We first prove that the total bicomplex completed with the embedding $A \hookrightarrow \mathrm{L}\left(\Upsilon_{00}, A\right)$ gives a free resolution of $A$.

By definition, each of the Polish $G$-modules $\mathrm{L}\left(\Upsilon_{p q}, A\right) \cong \mathrm{L}\left(G, \mathrm{~L}\left(\Gamma_{p q}, A\right)\right)$ is free. 
Consider now an arbitrary row in the bicomplex $\left(\mathrm{L}\left(\Upsilon_{p q}, A\right)\right)_{p, q \geq 0}$. Using the isomorphism of $\mathrm{L}\left(\Upsilon_{p, q+1}, A\right)$ with $\mathrm{L}\left(\Upsilon_{p q} \times G_{2}, A\right)$ through the identification of

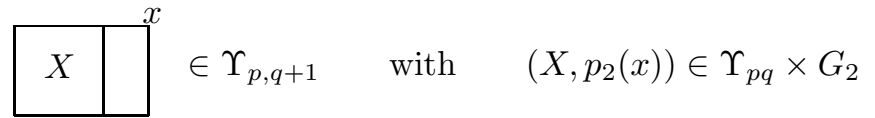

almost everywhere, we can write, for $F \in \mathrm{L}\left(\Upsilon_{p q}, A\right)$, that

$$
\left(\mathrm{d}^{\mathrm{h}} F\right)(X, s)=\mathrm{d}^{\mathrm{h}}(F(\cdot, s))(X)+(-1)^{q+1} F(X)
$$

almost everywhere. Hence, if $F \in \mathrm{L}\left(\Upsilon_{p q}, A\right)$ and $\mathrm{d}^{\mathrm{h}} F=0$, we can use the Fubini theorem to take an $s \in G_{2}$ such that $0=\mathrm{d}^{\mathrm{h}}(F(\cdot, s))(X)+(-1)^{q+1} F(X)$ for almost all $X \in \Upsilon_{p q}$. So, the horizontal cohomology of the bicomplex $\left(\mathrm{L}\left(\Upsilon_{p q}, A\right)\right)_{p, q \geq 0}$ vanishes. It follows that the the total cohomology is supported on the first column.

More precisely, this means that the total cohomology is the cohomology of the complex $T^{p}=\left\{F \in \mathrm{L}\left(\Upsilon_{p 0}, A\right) \mid \mathrm{d}^{\mathrm{h}} F=0\right\}$ with $\mathrm{d}^{\mathrm{v}}$ as a coboundary operator. Completing with $A \hookrightarrow \mathrm{L}\left(\Upsilon_{00}, A\right)$, we claim that we precisely get the standard resolution for the measurable cohomology of $G_{1}$ with coefficients in $A$. Observe that $\mathrm{L}\left(\Upsilon_{p 0}, A\right) \cong \mathrm{L}\left(G_{2} \times G_{1}^{p+1}, A\right)$ through the identification of the column vector $\left(x_{0}, \ldots, x_{p}\right)$ in $\Upsilon_{p 0}$ with $\left(p_{2}\left(x_{0}\right), p_{1}\left(x_{0}\right), \ldots, p_{1}\left(x_{p}\right)\right) \in G_{2} \times G_{1}^{p+1}$ almost everywhere. It is then easy to check that $T^{p} \cong \mathrm{L}\left(G_{1}^{p+1}, A\right)$ and that this isomorphism intertwines $\mathrm{d}^{\mathrm{v}}$ with the usual group coboundary operator. This proves our claim. So, we have proven that the total bicomplex of $\left(\mathrm{L}\left(\Upsilon_{p q}, A\right)\right)_{p, q \geq 0}$, completed with $A \hookrightarrow \mathrm{L}\left(\Upsilon_{00}, A\right)$ gives a free resolution of $A$. Hence, the measurable group cohomology $H(G, A)$ is given as the total cohomology of the bicomplex $\left(\mathrm{L}\left(\Upsilon_{p q}, A\right)^{G}\right)_{p, q \geq 0}$, which is precisely $\left(\mathrm{L}\left(\Gamma_{p q}, A\right)\right)_{p, q \geq 0}$.

Denote the total bicomplex of (17) by $D(A)=\left(D^{n}(A)\right)_{n \geq 0}$. We have proved that $H(D(A)) \cong H(G, A)$. But there is more. Since the cohomology $H(D, A)$ turns short exact sequences of coefficient modules in a natural way into short exact cohomology sequences, the cohomology theory $H(D, A)$ satisfies the Buchsbaum criterion. We can calculate $H(G, A)$ using the complex $\left(\mathcal{F}_{\text {Borel }}\left(G^{n}, A\right)\right)_{n \geq 0}$, where $\mathcal{F}_{\text {Borel }}$ denotes all Borel measurable functions and where the coboundary maps are defined in the Preliminaries. Now whenever $I:\left(\mathcal{F}_{\text {Borel }}\left(G^{n}, A\right)\right) \rightarrow\left(D^{n}(A)\right)$ is a natural cochain transformation, which is the identity on $\mathcal{F}_{\text {Borel }}\left(G^{0}, A\right)=A=D^{0}(A)$, we can conclude that this cochain transformation is a cohomology isomorphism. Such a cochain transformation can be written as

$$
I: \mathcal{F}_{\text {Borel }}\left(G^{n}, A\right) \rightarrow D^{n}(A): I(F)(X)=\sum_{\text {path in } X} \operatorname{Sign}(\text { path }) F(\text { path }),
$$

where $X \in \Gamma_{p q}, p+q=n$ and a path in e.g. $X \in \Gamma_{23}$ is a thing like

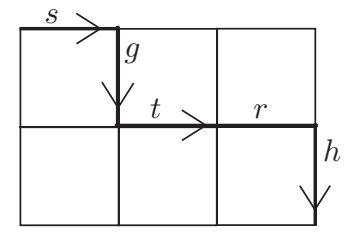

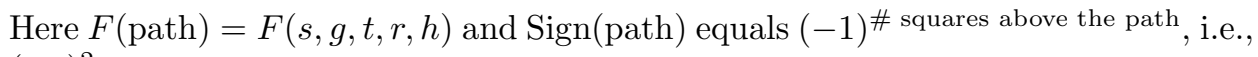
$(-1)^{2}=1$.

More formally, a path in $X \in \Gamma_{p q}$ is a path that starts in the top left corner of $X$, goes either down or right and ends, after $p+q=n$ steps in the bottom 
right corner of $X$. We evaluate $F \in \mathcal{F}_{\text {Borel }}\left(G^{n}, A\right)$ on the edges along the path of length $n$. The sign of the path is defined as -1 to the power the number of squares that are above the path. An elementary computation learns that $I$ is a cochain transformation.

Remark 4.2. We can also write a natural cochain transformation $I^{\prime}: D^{n}(A) \rightarrow$ $\mathrm{L}\left(G^{n}, A\right)$, yielding the inverse isomorphism $H(D(A)) \rightarrow H(G, A)$. Define the measure class isomorphism $G^{n} \rightarrow \Gamma_{n n}:\left(x_{1}, \ldots, x_{n}\right) \mapsto X\left(x_{1}, \ldots, x_{n}\right)$, where

$$
X\left(x_{1}\right)=x_{1}, \quad X\left(x_{1}, x_{2}\right)=\begin{array}{|l|l|}
\hline x_{1} & \\
\hline & x_{2} \\
\hline
\end{array}
$$

and where $X\left(x_{1}, \ldots, x_{n}\right) \in \Gamma_{n n}$ is defined analogously by putting $x_{1}, \ldots, x_{n}$ in boxes along the diagonal. Further, we define $P_{i}: \Gamma_{n n} \rightarrow \Gamma_{n-i, i}$, where $P_{i} X$ is the lower left corner of $X$, i.e. consisting of the $n-i$ final lines and $i$ first columns of $X$. We can now define

$$
I^{\prime}: D^{n}(A) \rightarrow \mathrm{L}\left(G^{n}, A\right): I^{\prime}(F)\left(x_{1}, \ldots, x_{n}\right)=\sum_{i=0}^{n} F\left(P_{i}\left(X\left(x_{1}, \ldots, x_{n}\right)\right)\right) .
$$

One verifies that $I^{\prime}$ is a cochain transformation.

Observe that $\Gamma_{0 n}=G_{2}^{n}$ and that $\mathrm{d}^{\mathrm{h}}: \mathrm{L}\left(\Gamma_{0 n}, A\right) \rightarrow \mathrm{L}\left(\Gamma_{0, n+1}, A\right)$ coincides with the usual coboundary operator $\mathrm{d}: \mathrm{L}\left(G_{2}^{n}, A\right) \rightarrow \mathrm{L}\left(G_{2}^{n+1}, A\right)$. Making the same observation for the first column of the bicomplex (17), we obtain a natural cochain transformation

$$
\begin{aligned}
& J:\left(D^{n}(A)\right)_{n} \rightarrow\left(K^{n}(A)\right)_{n}, \quad \text { where } \\
& D^{n}(A)=\bigoplus_{p+q=n} \mathrm{~L}\left(\Gamma_{p q}, A\right) \quad \text { and } \quad K^{n}(A)=\mathrm{L}\left(G_{1}^{n}, A\right) \oplus \mathrm{L}\left(G_{2}^{n}, A\right),
\end{aligned}
$$

and where we just have to explain that, for $n=0$, we take $A \ni a \mapsto a \oplus a \in A \oplus A$.

The following definition may seem a bit pedantic, but implies in a natural way how to define the Kac 0-cohomology (see Proposition 4.4 for the link with the cohomology of the cochain complex $\left(C^{n}(A)\right)$ defined in (15) and (16)).

Definition 4.3. The Kac cohomology $H($ m.p.,A) of the matched pair (m.p.) $G_{1}, G_{2} \subset G$ with coefficients in the Polish $G$-module $A$ is defined to be the cohomology of the mapping cone of the natural cochain transformation $J$ given by (19).

We recall that, by definition, the mapping cone $\left(M^{n}\right)_{n \geq-1}$ of the cochain transformation $J$ is defined by the formula

$$
M^{n}(A)=D^{n+1}(A) \oplus K^{n}(A) \quad \text { and } \quad \mathrm{d}(F, G)=(\mathrm{d} F,-\mathrm{d} G+J F) .
$$

We now explain how to complete the total bicomplex of (12) in order to obtain the Kac cohomology.

Proposition 4.4. Define, for $n \geq 1, C^{n}(A)$ as in (15). Define $C^{0}(A)=A$, $C^{-1}(A)=0$. Define

$$
\mathrm{d}: C^{0}(A) \rightarrow C^{1}(A):(\mathrm{d} a)\left(h \frac{s}{t} \frac{s}{t} g\right)=x \cdot a-s \cdot a-h \cdot a+a .
$$


This coboundary compiles with (16) to a cochain complex $\left(C^{n}(A)\right)_{n \geq-1}$. The inclusions $C^{n}(A) \hookrightarrow D^{n+1}(A)$, together with the map

$$
C^{0}(A) \rightarrow M^{0}(A)=\mathrm{L}\left(\Gamma_{10}, A\right) \oplus \mathrm{L}\left(\Gamma_{01}, A\right) \oplus A \oplus A: a \mapsto \mathrm{d}^{\mathrm{v}} a \oplus \mathrm{d}^{\mathrm{h}} a \oplus a \oplus a,
$$

define a natural cochain transformation $\left(C^{n}(A)\right) \rightarrow\left(M^{n}(A)\right)$ which is a cohomology isomorphism.

In particular, we conclude from Proposition 3.6 that the group of extensions of the matched pair $G_{1}, G_{2} \subset G$ is precisely the Kac 2-cohomology $H^{2}(m . p ., \mathbb{T})$ with coefficients in the trivial $G$-module $\mathbb{T}$.

Proof. For $n \geq 2, J: D^{n}(A) \rightarrow K^{n}(A)$ is surjective, with kernel the image of $C^{n-1}(A)$. This shows that the cochain transformation $\left(C^{n}(A)\right) \rightarrow\left(M^{n}(A)\right)$ is an isomorphism in $n$-cohomology for $n \geq 2$. For $n=0,1$, the same follows by an explicit verification.

The mapping cone of a cochain transformation is made for getting long exact sequences. So, the following is an immediate consequence of Proposition 4.1.

Corollary 4.5. The Kac cohomology H(m.p., A) of the matched pair $G_{1}, G_{2} \subset G$ with coefficients in the Polish $G$-module A satisfies the long exact sequence

$$
\begin{aligned}
0 & \rightarrow A^{G} \rightarrow A^{G_{1}} \oplus A^{G_{2}} \rightarrow H^{0}(m . p ., A) \rightarrow H^{1}(G, A) \rightarrow H^{1}\left(G_{1}, A\right) \oplus H^{1}\left(G_{2}, A\right) \\
& \rightarrow H^{1}(m . p ., A) \rightarrow H^{2}(G, A) \rightarrow H^{2}\left(G_{1}, A\right) \oplus H^{2}\left(G_{2}, A\right) \rightarrow H^{2}(m . p ., A) \\
& \rightarrow H^{3}(G, A) \rightarrow H^{3}\left(G_{1}, A\right) \oplus H^{3}\left(G_{2}, A\right) \rightarrow \cdots .
\end{aligned}
$$

Recall here that $H^{2}($ m.p., $\mathbb{T})$ is the group of extensions of the matched pair, by Propositions 3.6 and 4.4 .

Remark 4.6. Once we have, for a good class of locally compact groups $G$ and Polish $G$-modules $A$, a natural way to write a cochain complex $\left(E^{n}(G, A)\right)$ whose cohomology is the cohomology of $G$, we can expect that the Kac cohomology of a matched pair $G_{1}, G_{2} \subset G$ is the cohomology of the mapping cone of the cochain transformation $\left(E^{n}(G, A)\right)_{n} \rightarrow\left(E^{n}\left(G_{1}, A\right) \oplus E^{n}\left(G_{2}, A\right)\right)_{n}$. Concrete applications of this principle can be found below (see Proposition 7.1).

If $A$ is a Polish $G$-module, we define $\mathrm{L}(G, A)$ as a Polish $G$-module by writing $(x \cdot F)(y)=x \cdot F\left(x^{-1} y\right)$ for $F \in \mathrm{L}(G, A)$ and $x, y \in G$. Sending $a \in A$ to the constant function $a$, we get an embedding $A \hookrightarrow \mathrm{L}(G, A)$ of $A$ as a closed submodule of $\mathrm{L}(G, A)$. As a consequence of Corollary 4.5, we get the following result.

Corollary 4.7. If $G_{1}, G_{2} \subset G$ is a matched pair, then $H^{n}(m . p ., \mathrm{L}(G, A))=0$ for all $n \geq 1$ and all Polish $G$-modules A. In particular, the Kac cohomology satisfies the Buchsbaum criterion.

Proof. Since the group cohomology with coefficients in $\mathrm{L}(G, A)$ vanishes, this is an immediate consequence of the Kac exact sequence.

It is now possible to interpret the Kac cohomology of the matched pair $G_{1}, G_{2} \subset$ $G$ with coefficients in the Polish $G$-module $A$ as an ordinary group cohomology with coefficients in a well-chosen module. 
Corollary 4.8. Let $G_{1}, G_{2} \subset G$ be a matched pair and let $A$ be a Polish $G$-module. Then the Kac cohomology $H($ m.p., A) is the measurable group cohomology of $G$ with coefficients in the Polish G-module

$$
\frac{\mathrm{L}\left(G / G_{1} \sqcup G / G_{2}, A\right)}{A} .
$$

Proof. By Proposition 4.4 the Kac cohomology is defined by the total bicomplex of $\left(\mathrm{L}\left(\Gamma_{p q}, A\right)\right)_{p, q \geq 1}$ completed with $A \cong \mathrm{L}\left(\Gamma_{00}, A\right) \rightarrow \mathrm{L}\left(\Gamma_{11}, A\right): a \mapsto \mathrm{d}(a)=$ $\left(\mathrm{d}_{1}^{\mathrm{v}}-\mathrm{d}_{0}^{\mathrm{v}}\right)\left(\mathrm{d}_{1}^{\mathrm{h}}-\mathrm{d}_{0}^{\mathrm{h}}\right) a$. Now consider the $G$-equivariant analogue, which is the total bicomplex of $\left(\mathrm{L}\left(\Upsilon_{p q}, A\right)\right)_{p, q \geq 1}$ completed with $\mathrm{L}\left(\Upsilon_{00}, A\right) \rightarrow \mathrm{L}\left(\Upsilon_{11}, A\right): F \mapsto \mathrm{d} F=$ $\left(\mathrm{d}_{1}^{\mathrm{v}}-\mathrm{d}_{0}^{\mathrm{v}}\right)\left(\mathrm{d}_{1}^{\mathrm{h}}-\mathrm{d}_{0}^{\mathrm{h}}\right) F$. Denote the complex obtained as such by $\left(R^{n}\right)$.

We claim that

$$
\frac{\mathrm{L}\left(G / G_{1} \sqcup G / G_{2}, A\right)}{A} \stackrel{\theta}{\rightarrow} R^{0} \rightarrow R^{1} \rightarrow \cdots \quad \text { where } \quad \theta\left(H_{1} \oplus H_{2}\right)=H_{1}-H_{2}
$$

is a free resolution. The proof of this claim will complete the proof of the corollary.

By definition, each of the Polish $G$-modules $R^{n}$ is free. Moreover, the exactness of $R^{n-1} \rightarrow R^{n} \rightarrow R^{n+1}$ for $n \geq 1$ follows from Corollary 4.7 stating that $H^{n}(m . p ., \mathrm{L}(G, A))=0$. Also, $\theta$ is injective. If $H_{1} \in \mathrm{L}\left(G / G_{1}, A\right)$ and $H_{2} \in \mathrm{L}\left(G / G_{2}, A\right)$ are such that $\theta\left(H_{1} \oplus H_{2}\right)=0$, then $H_{1}=H_{2}=F$, where $F \in \mathrm{L}(G, A)$ is invariant under translations by both $G_{1}$ and $G_{2}$. Hence, $F$ is a constant function, proving the injectivity of $\theta$.

We have to show that the kernel of $\mathrm{d}: \mathrm{L}\left(\Upsilon_{00}, A\right) \rightarrow \mathrm{L}\left(\Upsilon_{11}, A\right)$ is the image of $\theta$. It is immediate that the image of $\theta$ is included in the kernel of d. Let $F \in \mathrm{L}\left(\Upsilon_{00}, A\right)$ and $\mathrm{d} F=0$. This means that

$$
F(x y)-F\left(x q_{2}(y)\right)-F\left(x p_{1}(y)\right)+F(x)=0 \quad \text { for almost all } \quad(x, y) \in G \times G .
$$

Using the Fubini theorem, we fix $x \in G$ such that the previous equation holds for almost all $y \in G$. Define $H_{1} \in \mathrm{L}\left(G / G_{1}, A\right)$ and $H_{2} \in \mathrm{L}\left(G / G_{2}, A\right)$ by the formulas $H_{1}(y)=F\left(x q_{2}(y)\right)$ and $H_{2}(y)=F(x)-F\left(x p_{1}(y)\right)$. Then, $F=\theta\left(H_{1} \oplus H_{2}\right)$.

Remark 4.9. Observe that it is clear that for any Polish $G$-module $A$ we have $H\left(G, \mathrm{~L}\left(G / G_{i}, A\right)\right)=H\left(G_{i}, A\right)$. Hence, the Kac exact sequence is exactly the long exact cohomology sequence that corresponds to the exact sequence of Polish $G$ modules

$$
0 \rightarrow A \rightarrow \mathrm{L}\left(G / G_{1} \sqcup G / G_{2}, A\right) \rightarrow \frac{\mathrm{L}\left(G / G_{1} \sqcup G / G_{2}, A\right)}{A} \rightarrow 0 .
$$

\section{Pentagonal Cohomology and isomorphism with KaC Cohomology}

In Definition 3.2 we defined the 2-cohomology group associated with a pentagonal transformation. Recall (14), where we defined face operators $\mathrm{d}_{i}^{\mathrm{h}}: \mathrm{L}\left(\Gamma_{p q}, A\right) \rightarrow$ $\mathrm{L}\left(\Gamma_{p, q+1}, A\right)$ and $\mathrm{d}_{j}^{\mathrm{v}}: \mathrm{L}\left(\Gamma_{p q}, A\right) \rightarrow \mathrm{L}\left(\Gamma_{p+1, q}, A\right)$ for $0 \leq i \leq q+1$ and $0 \leq j \leq p+1$. For convenience of notation, we write moreover $\mathrm{d}_{q+2}^{\mathrm{h}}=\mathrm{d}_{q+1}^{\mathrm{h}}$ and $\mathrm{d}_{-1}^{\mathrm{v}}=\mathrm{d}_{0}^{\mathrm{v}}$ on $\mathrm{L}\left(\Gamma_{p q}, A\right)$. Define then, for $n \geq 1$,

$$
\mathrm{d}_{\text {pent }}: \mathrm{L}\left(\Gamma_{n n}, A\right) \rightarrow \mathrm{L}\left(\Gamma_{n+1, n+1}, A\right): \mathrm{d}_{\text {pent }}=\sum_{i=0}^{n+2}(-1)^{i} \mathrm{~d}_{i}^{\mathrm{h}} \mathrm{d}_{i-1}^{\mathrm{v}} .
$$

In Remark 4.2 we defined the measure class isomorphism $G^{n} \rightarrow \Gamma_{n n}$. Using this, we can identify the usual complex for the measurable cohomology of $G$ with the 
complex defined by

$$
\mathrm{d}_{\text {group }}: \mathrm{L}\left(\Gamma_{n n}, A\right) \rightarrow \mathrm{L}\left(\Gamma_{n+1, n+1}, A\right): \mathrm{d}_{\text {group }}=\sum_{i=0}^{n+1}(-1)^{i} \mathrm{~d}_{i}^{\mathrm{h}} \mathrm{d}_{i}^{\mathrm{v}} .
$$

It is then clear that the injective map $\mathrm{d}_{n+1}^{\mathrm{h}} \mathrm{d}_{0}^{\mathrm{v}}: \mathrm{L}\left(\Gamma_{n n}, A\right) \rightarrow \mathrm{L}\left(\Gamma_{n+1, n+1}, A\right)$ intertwines $\mathrm{d}_{\text {pent }}$ and $\mathrm{d}_{\text {group. }}$. Hence, $\mathrm{d}_{\text {pent }}$ turns $E^{n}(A):=\mathrm{L}\left(\Gamma_{n n}, A\right)$ for $n \geq 1$ into a cochain complex. Remark that the 2-cohomology of this complex is, by definition, the 2-cohomology associated with the pentagonal transformation.

We can complete the cochain complex $\left(E^{n}(A)\right)_{n \geq 1}$, defining $E^{0}(A)=A \oplus A$ with

$$
\mathrm{d}_{\text {pent }}: A \oplus A \rightarrow \mathrm{L}\left(\Gamma_{11}, A\right): \mathrm{d}_{\text {pent }}(a, b)=\mathrm{d}(a)+\mathrm{d}_{0}^{\mathrm{h}} \mathrm{d}_{1}^{\mathrm{v}}(b),
$$

where $\mathrm{d}: A \rightarrow \mathrm{L}\left(\Gamma_{11}, A\right)$ is as in Proposition 4.4 .

Definition 5.1. Given a matched pair $G_{1}, G_{2} \subset G$, we define the pentagonal cohomology $H$ (pent, $A$ ) with coefficients in the Polish $G$-module $A$ as the cohomology of the cochain complex $\left(E^{n}(A)\right)_{n \geq 0}$ defined above.

Recall the Kac cochain complex $\left(C^{n}(A)\right)$ that we defined in (15) and Proposition 4.4. Using Remark 4.2 and the cochain transformation $I^{\prime}: D^{n}(A) \rightarrow \mathrm{L}\left(G^{n}, A\right) \cong$ $\mathrm{L}\left(\Gamma_{n n}, A\right)$ defined there, we get a cochain transformation $T: C^{n}(A) \rightarrow E^{n}(A)$, which is defined such that for $n \geq 1$, the diagram

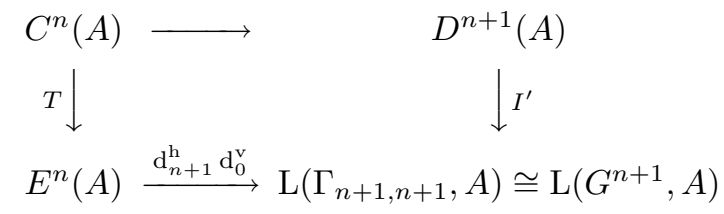

commutes. For $n=0$, we put $T: C^{0}(A)=A \rightarrow A \oplus A=E^{0}(A): a \mapsto a \oplus 0$.

We prove now that $T$ is a cohomology isomorphism. Hence, the pentagonal and Kac cohomologies are isomorphic.

Proposition 5.2. Let $G_{1}, G_{2} \subset G$ be a matched pair. The cochain transformation $T$ is a cohomology isomorphism between Kac cohomology and pentagonal cohomology.

Proof. As in (13), we can write equivariant face operators

$$
\begin{array}{lll}
\mathrm{d}_{i}^{\mathrm{h}}: \mathrm{L}\left(\Upsilon_{p q}, A\right) \rightarrow \mathrm{L}\left(\Upsilon_{p, q+1}, A\right) & \text { for } \quad 0 \leq i \leq q+1 \\
\mathrm{~d}_{j}^{\mathrm{v}}: \mathrm{L}\left(\Upsilon_{p q}, A\right) \rightarrow \mathrm{L}\left(\Upsilon_{p+1, q}, A\right) & \text { for } \quad 0 \leq j \leq p+1 .
\end{array} \quad \text { and }
$$

For convenience of notation, we put moreover $\mathrm{d}_{q+2}^{\mathrm{h}}=\mathrm{d}_{q+1}^{\mathrm{h}}$ and $\mathrm{d}_{-1}^{\mathrm{v}}=\mathrm{d}_{0}^{\mathrm{v}}$ on $\mathrm{L}\left(\Upsilon_{p q}, A\right)$. Using this notation, we get the $G$-equivariant cochain complex $\left(P^{n}(A)\right)$ such that for $n \geq 1, P^{n}(A)=\mathrm{L}\left(\Upsilon_{n n}, A\right)$ and

$$
\mathrm{d}_{\text {pent }}: \mathrm{L}\left(\Upsilon_{n n}, A\right) \rightarrow \mathrm{L}\left(\Upsilon_{n+1, n+1}, A\right): \mathrm{d}_{\text {pent }}=\sum_{i=0}^{n+2}(-1)^{i} \mathrm{~d}_{i}^{\mathrm{h}} \mathrm{d}_{i-1}^{\mathrm{v}} .
$$

Also, write $P^{0}=\mathrm{L}\left(\Upsilon_{00}, A\right) \oplus \mathrm{L}\left(\Upsilon_{00}, A\right)$ and

$$
\begin{aligned}
& \mathrm{d}_{\text {pent }}: \mathrm{L}\left(\Upsilon_{00}, A\right) \oplus \mathrm{L}\left(\Upsilon_{00}, A\right) \rightarrow \mathrm{L}\left(\Upsilon_{11}, A\right): \\
& \mathrm{d}_{\text {pent }}(\alpha \oplus \beta)=\left(\mathrm{d}_{0}^{\mathrm{h}}-\mathrm{d}_{1}^{\mathrm{h}}\right)\left(\mathrm{d}_{0}^{\mathrm{v}}-\mathrm{d}_{1}^{\mathrm{v}}\right) \alpha+\mathrm{d}_{0}^{\mathrm{h}} \mathrm{d}_{1}^{\mathrm{v}} \beta .
\end{aligned}
$$


Then, the cochain complex $\left(E^{n}(A)\right)$ of the pentagonal cohomology consists precisely of the fixed points under $G$ in $\left(P^{n}(A)\right)$.

We now complete the above $G$-equivariant complex by $\frac{\mathrm{L}\left(G / G_{1} \sqcup G / G_{2}, A\right)}{A}$ in order to get a resolution. Using Corollary 4.8, it is sufficient to prove that

$$
\frac{\mathrm{L}\left(G / G_{1} \sqcup G / G_{2}, A\right)}{A} \stackrel{\pi}{\rightarrow} P^{0}(A) \rightarrow P^{1}(A) \rightarrow \cdots,
$$

with $\pi\left(H_{1} \oplus H_{2}\right)=\left(H_{1}-H_{2}\right) \oplus 0$, is a free resolution of $\frac{\mathrm{L}\left(G / G_{1} \sqcup G / G_{2}, A\right)}{A}$. Indeed, we then know that the pentagonal cohomology is isomorphic with the Kac cohomology and that the natural cochain transformation $T$ induces a cohomology isomorphism.

From the lemma following this proposition, we know that the sequence $P^{0}(A) \rightarrow$ $P^{1}(A) \rightarrow \cdots$ is exact. It remains to prove that if $F \oplus G \in P^{0}(A)$ and $\mathrm{d} F+\mathrm{d}_{0}^{\mathrm{h}} \mathrm{d}_{1}^{\mathrm{v}} G=$ 0 , then $F \oplus G$ belongs to the image of $\pi$. From the proof of Corollary 4.8 it follows that it is sufficient to prove that $G=0$. But, $\left(\mathrm{d}_{1}^{\mathrm{v}}-\mathrm{d}_{0}^{\mathrm{v}}\right)\left(\mathrm{d}_{1}^{\mathrm{h}}-\mathrm{d}_{0}^{\mathrm{h}}\right) F+\mathrm{d}_{0}^{\mathrm{h}} \mathrm{d}_{1}^{\mathrm{v}} G=0$. Apply $\mathrm{d}^{\mathrm{h}}=\mathrm{d}_{0}^{\mathrm{h}}-\mathrm{d}_{1}^{\mathrm{h}}+\mathrm{d}_{2}^{\mathrm{h}}$ to both sides of this last equation. We find that $0=$ $\mathrm{d}_{2}^{\mathrm{h}} \mathrm{d}_{0}^{\mathrm{h}} \mathrm{d}_{1}^{\mathrm{v}} G$ and hence, $G=0$.

Lemma 5.3. Let $G_{1}, G_{2} \subset G$ be a matched pair and let $A$ be a Polish $G$-module. The sequence

$$
P^{0}(A) \rightarrow P^{1}(A) \rightarrow \cdots,
$$

constructed in the proof of the previous proposition, is exact.

Proof. We temporarily consider some cohomology which is intermediate between the pentagonal cohomology and the group cohomology of $G$. For $n \geq 0$, we define

$$
\mathrm{d}_{\text {temp }}: \mathrm{L}\left(\Upsilon_{n+1, n}, A\right) \rightarrow \mathrm{L}\left(\Upsilon_{n+2, n+1}, A\right): \mathrm{d}_{\text {temp }}=\sum_{i=0}^{n+2}(-1)^{i} \mathrm{~d}_{i}^{\mathrm{h}} \mathrm{d}_{i}^{\mathrm{v}} .
$$

The injective maps

$$
\mathrm{L}\left(\Upsilon_{n n}, A\right) \stackrel{\mathrm{d}_{0}^{\mathrm{v}}}{\longrightarrow} \mathrm{L}\left(\Upsilon_{n+1, n}, A\right) \stackrel{\mathrm{d}_{n+1}^{\mathrm{h}}}{\longrightarrow} \mathrm{L}\left(\Upsilon_{n+1, n+1}, A\right)
$$

intertwine $\mathrm{d}_{\text {pent }}, \mathrm{d}_{\text {temp }}$ and $\mathrm{d}_{\text {group }}$ for $n \geq 1$.

Suppose $n \geq 1$ and write $\mathrm{d}_{\text {temp }}^{n}$ for a while. We identify $\mathrm{L}\left(\Upsilon_{n+2, n+1}, A\right)$ with $\mathrm{L}\left(G \times \Upsilon_{n+1, n}, A\right)$ through the identification of

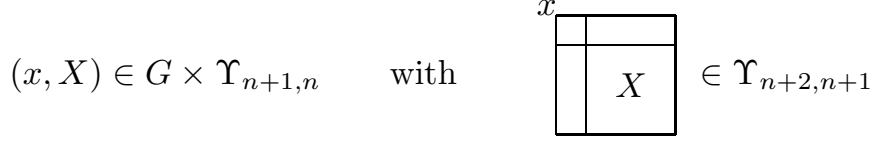

almost everywhere. Observe that $\left(\mathrm{d}_{\text {temp }}^{n} F\right)(x, X)=F(X)-\mathrm{d}_{\text {temp }}^{n-1}(F(x, \cdot))(X)$ for almost all $(x, X) \in G \times \Upsilon_{n+1, n}$. Now if $F \in \mathrm{L}\left(\Upsilon_{n+1, n}, A\right)$ and $\mathrm{d}_{\text {temp }}^{n} F=0$, we take a Borel measurable representative for $F$ that we still denote by $F$. Then,

$$
F(X)=\mathrm{d}_{\text {temp }}^{n-1}(F(x, \cdot))(X) \text { for almost all }(x, X) \in G \times \Upsilon_{n+1, n} .
$$

By the Fubini theorem, we can take an $x \in G$ such that the previous equation holds for almost all $X \in \Upsilon_{n+1, n}$. So, we conclude that $F$ is a coboundary.

As such, we did not only prove that the cohomology of $d_{\text {temp }}$ is trivial, but we also proved the following: if $\theta: A \rightarrow B$ is a continuous $G$-equivariant homomorphism between the Polish $G$-modules $A$ and $B$ and if $F \in \mathrm{L}\left(\Upsilon_{n+1, n}, A\right)$ such that $\mathrm{d}_{\text {temp }}(\theta(F))=0$, then there exists $K \in \mathrm{L}\left(\Upsilon_{n, n-1}, A\right)$ such that $\theta(F)=\theta\left(\mathrm{d}_{\text {temp }} K\right)$. 


\section{Consider}

$$
\mathrm{d}_{\text {temp }}: \mathrm{L}\left(G_{1}, A\right) \rightarrow \mathrm{L}\left(\Upsilon_{10}, A\right):\left(\mathrm{d}_{\text {temp }} F\right)\left(\left.\right|_{y} ^{x}\right)=F\left(p_{1}(x)\right)-F\left(p_{1}(y)\right) .
$$

Now if $F \in \mathrm{L}\left(\Upsilon_{10}, A\right)$ and $\mathrm{d}_{\text {temp }} F=0$, there exists $K \in \mathrm{L}\left(G_{1}, A\right)$ such that $F=\mathrm{d}_{\text {temp }} K$. The same kind of statement involving $\theta: A \rightarrow B$ as above also holds.

We now prove the exactness of the sequence $P^{0}(A) \rightarrow P^{1}(A) \rightarrow \cdots$. Let $n \geq$ 1 and $\alpha \in \mathrm{L}\left(\Upsilon_{n n}, A\right)$ such that $\mathrm{d}_{\text {pent }} \alpha=0$. Then, $\mathrm{d}_{0}^{\mathrm{v}} \alpha \in \mathrm{L}\left(\Upsilon_{n+1, n}, A\right)$ and $\mathrm{d}_{\text {temp }} \mathrm{d}_{0}^{\mathrm{v}} \alpha=0$. Using the results above, we can take $\beta \in \mathrm{L}\left(\Upsilon_{n, n-1}, A\right)$ such that $\mathrm{d}_{0}^{\mathrm{v}} \alpha=\mathrm{d}_{\text {temp }} \beta$.

Suppose now first that $n \geq 2$. Applying $\mathrm{d}_{0}^{\mathrm{v}}-\mathrm{d}_{1}^{\mathrm{v}}$ to both sides of this equation yields

$$
0=\sum_{i=1}^{n+1}(-1)^{i} \mathrm{~d}_{i}^{\mathrm{h}} \mathrm{d}_{i+1}^{\mathrm{v}}\left(\mathrm{d}_{0}^{\mathrm{v}}-\mathrm{d}_{1}^{\mathrm{v}}\right) \beta
$$

We now use the identification of $\mathrm{L}\left(\Upsilon_{n+1, n-1}, A\right)$ with $\mathrm{L}\left(\Upsilon_{n-1, n-2}, \mathrm{~L}\left(\Upsilon_{10}, A\right)\right)$ by identifying

$$
(X, Y) \in \Upsilon_{n-1, n-2} \times \Upsilon_{10} \quad \text { with } \quad X \Upsilon_{n+1, n-1}
$$

almost everywhere. We also identify $\mathrm{L}\left(\Upsilon_{n, n-1}, A\right)$ with $\mathrm{L}\left(\Upsilon_{n-1, n-2}, \mathrm{~L}\left(\Upsilon_{00}, A\right)\right)$ as above. We consider $\theta:=\mathrm{d}_{0}^{\mathrm{v}}-\mathrm{d}_{1}^{\mathrm{v}}: \mathrm{L}\left(\Upsilon_{00}, A\right) \rightarrow \mathrm{L}\left(\Upsilon_{10}, A\right)$ as a morphism of Polish modules. Using all these identifications, (21) becomes $\mathrm{d}_{\text {temp }}(\theta(\beta))=0$. From the results in the beginning of the proof, we find $\gamma \in \mathrm{L}\left(\Upsilon_{n-2, n-3}, \mathrm{~L}\left(\Upsilon_{00}, A\right)\right)$ such that $\theta(\beta)=\theta\left(\mathrm{d}_{\text {temp }} \gamma\right)$. Re-identifying, we have found $\gamma \in \mathrm{L}\left(\Upsilon_{n-1, n-2}, A\right)$ such that

$$
\left(\mathrm{d}_{0}^{\mathrm{v}}-\mathrm{d}_{1}^{\mathrm{v}}\right) \beta=\sum_{i=1}^{n}(-1)^{i} \mathrm{~d}_{i}^{\mathrm{h}} \mathrm{d}_{i+1}^{\mathrm{v}}\left(\mathrm{d}_{0}^{\mathrm{v}}-\mathrm{d}_{1}^{\mathrm{v}}\right) \gamma
$$

and hence,

$$
\left(\mathrm{d}_{0}^{\mathrm{v}}-\mathrm{d}_{1}^{\mathrm{v}}\right) \beta=\left(\mathrm{d}_{0}^{\mathrm{v}}-\mathrm{d}_{1}^{\mathrm{v}}\right) \mathrm{d}_{\text {temp }} \gamma .
$$

Observe that the argument works well for $n=2$ by using (20).

Write $\tilde{\beta}=\beta-\mathrm{d}_{\text {temp }} \gamma$. Then $\mathrm{d}_{0}^{\mathrm{v}} \alpha=\mathrm{d}_{\text {temp }} \beta=\mathrm{d}_{\text {temp }} \tilde{\beta}$ and moreover $\left(\mathrm{d}_{0}^{\mathrm{v}}-\mathrm{d}_{1}^{\mathrm{v}}\right) \tilde{\beta}$ $=0$. But then, there is a unique $\mu \in \mathrm{L}\left(\Upsilon_{n-1, n-1}, A\right)$ such that $\tilde{\beta}=\mathrm{d}_{0}^{\mathrm{v}} \mu$. We conclude that $\mathrm{d}_{0}^{\mathrm{v}} \alpha=\mathrm{d}_{\text {temp }} \mathrm{d}_{0}^{\mathrm{V}} \mu=\mathrm{d}_{0}^{\mathrm{v}} \mathrm{d}_{\text {pent }} \mu$ and hence, $\alpha=\mathrm{d}_{\text {pent }} \mu$.

Finally, consider the case $n=1$. So, we have $\alpha \in \mathrm{L}\left(\Upsilon_{11}, A\right)$ such that $\mathrm{d}_{\text {pent }} \alpha=0$. The beginning of the argument above works well and we find $\beta \in \mathrm{L}\left(\Upsilon_{10}, A\right)$ such that

$$
\mathrm{d}_{0}^{\mathrm{v}} \alpha=\left(\mathrm{d}_{0}^{\mathrm{h}} \mathrm{d}_{0}^{\mathrm{v}}-\mathrm{d}_{1}^{\mathrm{h}} \mathrm{d}_{1}^{\mathrm{v}}+\mathrm{d}_{1}^{\mathrm{h}} \mathrm{d}_{2}^{\mathrm{v}}\right) \beta .
$$

Apply $\mathrm{d}_{0}^{\mathrm{v}}-\mathrm{d}_{1}^{\mathrm{v}}$ to both sides and conclude that $\mathrm{d}_{1}^{\mathrm{h}}\left(\mathrm{d}_{0}^{\mathrm{v}}-\mathrm{d}_{1}^{\mathrm{v}}\right)\left(\mathrm{d}_{1}^{\mathrm{v}}-\mathrm{d}_{2}^{\mathrm{v}}\right) \beta=0$. Hence, $\left(\mathrm{d}_{2}^{\mathrm{v}}-\mathrm{d}_{3}^{\mathrm{v}}\right)\left(\mathrm{d}_{0}^{\mathrm{v}}-\mathrm{d}_{1}^{\mathrm{v}}\right) \beta=0$. So, we can take $\gamma \in \mathrm{L}\left(\Upsilon_{10}, A\right)$ such that

$$
\left(\mathrm{d}_{0}^{\mathrm{v}}-\mathrm{d}_{1}^{\mathrm{v}}\right) \beta=\mathrm{d}_{2}^{\mathrm{v}} \gamma \text {. }
$$

It follows that $\mathrm{d}^{\mathrm{v}} \beta=\mathrm{d}_{2}^{\mathrm{v}}(\gamma+\beta)$ and hence $\mathrm{d}^{\mathrm{v}} \mathrm{d}_{2}^{\mathrm{v}}(\gamma+\beta)=0$. This can be rewritten as $\mathrm{d}_{3}^{\mathrm{v}}\left(\mathrm{d}_{0}^{\mathrm{v}}-\mathrm{d}_{1}^{\mathrm{v}}\right)(\gamma+\beta)=0$ and hence, $\left(\mathrm{d}_{0}^{\mathrm{v}}-\mathrm{d}_{1}^{\mathrm{v}}\right)(\gamma+\beta)=0$. Combining this last equation with (22), we find that $\mathrm{d}^{\mathrm{v}} \gamma=0$. It is by now easy to find $\mu \in \mathrm{L}\left(\Upsilon_{00}, A\right)$ such that $\gamma=\mathrm{d}^{\mathrm{v}} \mu$ and hence, $\gamma=\left(\mathrm{d}_{0}^{\mathrm{v}}-\mathrm{d}_{1}^{\mathrm{v}}\right) \mu$. Write $\tilde{\beta}=\beta-\mathrm{d}_{1}^{\mathrm{v}} \mu$. Then,

$$
\left(\mathrm{d}_{0}^{\mathrm{v}}-\mathrm{d}_{1}^{\mathrm{v}}\right) \tilde{\beta}=\left(\mathrm{d}_{0}^{\mathrm{v}}-\mathrm{d}_{1}^{\mathrm{v}}\right)(\beta+\gamma)-\left(\mathrm{d}_{0}^{\mathrm{v}}-\mathrm{d}_{1}^{\mathrm{v}}\right)\left(\gamma+\mathrm{d}_{1}^{\mathrm{v}} \mu\right)=0-\left(\mathrm{d}_{0}^{\mathrm{v}}-\mathrm{d}_{1}^{\mathrm{v}}\right) \mathrm{d}_{0}^{\mathrm{v}} \mu=0 .
$$


So, we can take $\eta \in \mathrm{L}\left(\Upsilon_{00}, A\right)$ such that $\tilde{\beta}=\mathrm{d}_{0}^{\mathrm{v}} \eta$. Finally,

$$
\mathrm{d}_{\text {temp }} \tilde{\beta}=\mathrm{d}_{\text {temp }} \beta-\left(\mathrm{d}_{0}^{\mathrm{h}} \mathrm{d}_{0}^{\mathrm{v}}-\mathrm{d}_{1}^{\mathrm{h}}\left(\mathrm{d}_{1}^{\mathrm{v}}-\mathrm{d}_{2}^{\mathrm{v}}\right)\right) \mathrm{d}_{1}^{\mathrm{v}} \mu=\mathrm{d}_{0}^{\mathrm{v}}\left(\alpha-\mathrm{d}_{0}^{\mathrm{h}} \mathrm{d}_{1}^{\mathrm{v}} \mu\right) .
$$

Hence,

$$
\left(\mathrm{d}_{0}^{\mathrm{h}} \mathrm{d}_{0}^{\mathrm{v}}-\mathrm{d}_{1}^{\mathrm{h}} \mathrm{d}_{0}^{\mathrm{v}}+\mathrm{d}_{1}^{\mathrm{h}} \mathrm{d}_{1}^{\mathrm{v}}\right)(\eta)=\alpha-\mathrm{d}_{0}^{\mathrm{h}} \mathrm{d}_{1}^{\mathrm{v}} \mu
$$

and so, $\alpha=d(\eta)+\mathrm{d}_{0}^{\mathrm{h}} \mathrm{d}_{1}^{\mathrm{v}}(\eta+\mu)=\mathrm{d}_{\text {pent }}(\eta \oplus(\eta+\mu))$.

\section{INTERPRETATION OF 1-COHOMOLOGY}

Fix a matched pair $G_{1}, G_{2} \subset G$. We give a natural interpretation of the Kac 1-cohomology $H^{1}($ m.p., $\mathbb{T})$ with coefficients in the trivial $G$-module $\mathbb{T}$.

As we explained in Subsection 3.1, the elements of $H^{2}(m . p ., \mathbb{T})$ can be interpreted as extensions $(M, \Delta)$

$$
e \longrightarrow\left(\mathrm{L}^{\infty}\left(G_{1}\right), \Delta_{1}\right) \longrightarrow(M, \Delta) \longrightarrow\left(\mathcal{L}\left(G_{2}\right), \hat{\Delta}_{2}\right) \longrightarrow e
$$

in the category of locally compact quantum groups. In particular, the element $0 \in H^{2}(m . p ., \mathbb{T})$ gives rise to an extension $(M, \Delta)$ that we describe explicitly as follows. It is called the bicrossed product of $G_{1}, G_{2}$.

The von Neumann algebra $M$ is the crossed product $M=G_{2} \ltimes \mathrm{L}^{\infty}\left(G / G_{2}\right)$ that we realize explicitly as follows. Identifying $\mathrm{L}^{\infty}\left(G / G_{2}\right)$ with $\mathrm{L}^{\infty}\left(G_{1}\right)$, we consider the Hilbert space $H=L^{2}\left(G_{2} \times G_{1}\right)$ and write

$$
\begin{aligned}
& \pi_{1}: \mathrm{L}^{\infty}\left(G_{1}\right) \rightarrow \mathrm{B}(H):\left(\pi_{1}(F) \xi\right)(s, g)=F\left(p_{1}(s g)\right) \xi(s, g), \\
& \lambda: \mathcal{L}\left(G_{2}\right) \rightarrow \mathrm{B}(H): \lambda(a)=a \otimes 1 .
\end{aligned}
$$

The von Neumann algebra $M$ is generated by $\pi_{1}\left(\mathrm{~L}^{\infty}\left(G_{1}\right)\right)$ and $\lambda\left(\mathcal{L}\left(G_{2}\right)\right)$ in $\mathrm{B}(H)$. The coproduct $\Delta: M \rightarrow M \otimes M$ on $M$ is then given by

$$
\begin{aligned}
& \Delta \pi_{1}=\left(\pi_{1} \otimes \pi_{1}\right) \Delta_{1}, \quad \text { where } \quad \Delta_{1}(F)(g, h)=F(g h) \quad \text { for all } g, h \in G_{1}, \\
& \Delta\left(\lambda_{s} \otimes 1\right)=\left(\left(\lambda_{s} \otimes 1 \otimes 1\right)\left(\pi_{1} \otimes \iota\right)\left(X_{s}\right)\right) \otimes 1, \\
& \text { where } \quad X_{s} \in \mathrm{L}^{\infty}\left(G_{1}\right) \otimes \mathcal{L}\left(G_{2}\right) \quad \text { is given by } \quad X_{s}(g)=\lambda_{p_{2}(s g)} .
\end{aligned}
$$

Since $M$ is a crossed product, there is a dual coaction

$$
\theta: M \rightarrow \mathcal{L}\left(G_{2}\right) \otimes M:\left\{\begin{array}{l}
\theta\left(\pi_{1}(F)\right)=1 \otimes \pi_{1}(F), \\
\theta\left(\lambda_{s} \otimes 1\right)=\lambda_{s} \otimes\left(\lambda_{s} \otimes 1\right) .
\end{array}\right.
$$

If we write somehow formally the morphism $\pi_{2}: M \rightarrow \mathcal{L}\left(G_{2}\right)$, we can consider that $\theta=\left(\pi_{2} \otimes \iota\right) \Delta$. This is explained in detail after Proposition 3.1 in [23].

Definition 6.1. A (left) coideal $I$ of a locally compact quantum group $(M, \Delta)$ is a von Neumann subalgebra $I \subset M$ satisfying $\Delta(I) \subset M \otimes I$.

We only work with left coideals, and so we leave out 'left' from now on. We shall give a bijective correspondence between the Kac 1-cohomology $H^{1}(m . p ., \mathbb{T})$ and a special class of coideals in the bicrossed product $(M, \Delta)$. If $I$ is a coideal, then $\theta(I) \subset \mathcal{L}\left(G_{2}\right) \otimes I$. So, $\left(\mathcal{L}\left(G_{2}\right), \hat{\Delta}_{2}\right)$ coacts on $I$. As such, $I$ is a $\mathcal{L}\left(G_{2}\right)$-comodule.

Definition 6.2. We say that a coideal $I$ of the bicrossed product $(M, \Delta)$ is full, if for every $s \in G_{2}$,

$$
E_{s}:=\left\{x \in I \mid \theta(x)=\lambda_{s} \otimes x\right\}
$$

is a one-dimensional subspace of $I$. 
We then have the following result.

Proposition 6.3. Let I be a coideal of $(M, \Delta)$. Then, the following are equivalent:

(1) I is full.

(2) There exists an isomorphism $\mu: \mathcal{L}\left(G_{2}\right) \rightarrow I$ of von Neumann algebras satisfying $(\iota \otimes \mu) \hat{\Delta}_{2}=\theta \mu$.

(3) There exists an $\mathcal{R} \in H^{1}(m . p ., \mathbb{T})$ such that

$$
I=\mathcal{R}\left(\mathcal{L}\left(G_{2}\right) \otimes 1\right) \mathcal{R}^{*},
$$

where we consider $\mathcal{R}: G_{2} \times G_{1} \rightarrow \mathbb{T}$ as a unitary multiplication operator on $\mathrm{L}^{2}\left(G_{2} \times G_{1}\right)$.

Moreover, the correspondence $\mathcal{R} \mapsto \mathcal{R}\left(\mathcal{L}\left(G_{2}\right) \otimes 1\right) \mathcal{R}^{*}$ defines a bijection between $H^{1}(m . p ., \mathbb{T})$ and the set of full coideals of the bicrossed product $(M, \Delta)$.

Proof. Recall that $H^{1}(m . p ., \mathbb{T})$ consists of the functions $\mathcal{R} \in \mathrm{L}\left(G_{2} \times G_{1}, \mathbb{T}\right)$ such that

$$
\overline{\mathcal{R}}\left(p_{2}(s g), h\right) \mathcal{R}(s, g h) \overline{\mathcal{R}}(s, g)=1 \quad \text { and } \quad \mathcal{R}(t, g) \overline{\mathcal{R}}(s t, g) \mathcal{R}\left(s, p_{1}(t g)\right)=1
$$

for almost all $(s, t, g, h) \in G_{2}^{2} \times G_{1}^{2}$.

Let $\mathcal{R}$ be such a function and consider $\mathcal{R}$ as a unitary (multiplication) operator on $\mathrm{L}^{2}\left(G_{2} \times G_{1}\right)$. Define

$$
I=\mathcal{R}\left(\mathcal{L}\left(G_{2}\right) \otimes 1\right) \mathcal{R}^{*} .
$$

Observe that $I$ is a von Neumann algebra generated by $\mathcal{R}\left(\lambda_{s} \otimes 1\right) \mathcal{R}^{*}$ for $s \in G_{2}$. Using the second equation satisfied by $\mathcal{R}$, one gets

$$
\mathcal{R}\left(\lambda_{s} \otimes 1\right) \mathcal{R}^{*}=\left(\lambda_{s} \otimes 1\right) \pi_{1}(\mathcal{R}(s, \cdot)) .
$$

Hence $I$ is a von Neumann subalgebra of $M$. Using the first relation satisfied by $\mathcal{R}$, one verifies immediately that $I$ is a coideal of $(M, \Delta)$. We also observe that $\mu: \mathcal{L}\left(G_{2}\right) \rightarrow I: \mu(a)=\mathcal{R}(a \otimes 1) \mathcal{R}^{*}$ defines an isomorphism of von Neumann algebras satisfying $(\iota \otimes \mu) \hat{\Delta}_{2}=\theta \mu$. Hence, $E_{s}=\mu\left(\mathbb{C} \lambda_{s}\right)$ and $I$ is full. So, we have proven that there is a map from $H^{1}(m . p ., \mathbb{T})$ to the set of coideals satisfying all three conditions in the statement of the proposition.

We prove that this map is injective. For this, it is sufficient to prove that, whenever $\mathcal{R} \in H^{1}($ m.p., $\mathbb{T})$ and $\mathcal{R}\left(\mathcal{L}\left(G_{2}\right) \otimes 1\right) \mathcal{R}^{*}=\mathcal{L}\left(G_{2}\right) \otimes 1$, then $\mathcal{R}=1$. In that case, $\left(\lambda_{s} \otimes 1\right) \pi_{1}(\mathcal{R}(s, \cdot)) \in \mathcal{L}\left(G_{2}\right) \otimes 1$ for all $s \in G_{2}$. Hence, $\pi_{1}(\mathcal{R}(s, \cdot)) \in \mathbb{C}$ and we find a function $\mathcal{V}: G_{2} \rightarrow \mathbb{T}$ such that $\mathcal{R}(s, g)=\mathcal{V}(s)$ for almost all $(s, g) \in G_{2} \times G_{1}$. Considering $\mathcal{R} \in \mathrm{L}\left(\Gamma_{11}, \mathbb{T}\right)$ and $\mathcal{V} \in \mathrm{L}\left(\Gamma_{01}, \mathbb{T}\right)$, this means that $\mathcal{R}=\mathrm{d}_{1}^{\mathrm{v}} \mathcal{V}$. Then, shifting to additive notation

$$
0=\mathrm{d}^{\mathrm{v}} \mathcal{R}=\left(\mathrm{d}_{0}^{\mathrm{v}}-\mathrm{d}_{1}^{\mathrm{v}}+\mathrm{d}_{2}^{\mathrm{v}}\right) \mathrm{d}_{1}^{\mathrm{v}} \mathcal{V}=\mathrm{d}_{0}^{\mathrm{v}} \mathrm{d}_{1}^{\mathrm{v}} \mathcal{V}
$$

This implies that $\mathcal{V}$ is trivial and hence $\mathcal{R}=0$ in $H^{1}(m . p ., \mathbb{T})$.

Suppose next that $I$ is a full coideal in the bicrossed product $(M, \Delta)$. We shall produce an element in $H^{1}(m . p ., \mathbb{T})$ such that $I$ is given by (23). Hence, $I$ will also satisfy the second statement of the proposition.

We claim that there exists a Borel measurable map $Y: G_{2} \rightarrow \mathcal{U}(I)$ such that $\theta(Y(s))=\lambda_{s} \otimes Y(s)$. Here, $\mathcal{U}(I)$ denotes the unitary group of $I$ equipped with the strong topology. As such, $\mathcal{U}(I)$ is a Polish group. Let $s \in G_{2}$ and $x \in E_{s}$. Then, 
$x^{*} x, x x^{*} \in E_{e}$. Further, $\mathbb{C} 1 \subset E_{e}$ implying that $E_{e}=\mathbb{C} 1$. This means that $E_{s}$ is of the form $\mathbb{C} u$, with $u$ a unitary in $I$. Define the Polish group $K \subset G_{2} \times \mathcal{U}(I)$ as

$$
K=\left\{(s, u) \in G_{2} \times \mathcal{U}(I) \mid \theta(u)=\lambda_{s} \otimes u\right\} .
$$

Define $\pi: K \rightarrow G_{2}: \pi(s, u)=s$. Then, $\pi$ is continuous and surjective. It follows that there exists a Borel section $G_{2} \rightarrow K$, and this proves our claim.

Denote by $M^{\theta}$ the subalgebra of $M$ consisting of $x \in M$ satisfying $\theta(x)=1 \otimes x$. Observe that, since $\theta$ is the dual coaction on the crossed product $G_{2} \ltimes \mathrm{L}^{\infty}\left(G_{1}\right)$, we get that $M^{\theta}=\pi_{1}\left(\mathrm{~L}^{\infty}\left(G_{1}\right)\right)$. For any $s \in G_{2},\left(\lambda_{s}^{*} \otimes 1\right) Y(s) \in M^{\theta}$. Hence, we can take $\mathcal{R}: G_{2} \times G_{1} \rightarrow \mathbb{T}$ Borel measurable such that $Y(s)=\left(\lambda_{s} \otimes 1\right) \pi_{1}(\mathcal{R}(s, \cdot))$ for all $s \in G_{2}$.

Since $I$ is a von Neumann algebra, we have $Y(s t)^{*} Y(s) Y(t) \in I \cap M^{\theta}=\mathbb{C}$. It follows that there is a measurable function $\mathcal{V}: G_{2} \times G_{1} \rightarrow \mathbb{T}$ such that

$$
\overline{\mathcal{R}(s t, g)} \mathcal{R}\left(s, p_{1}(t g)\right) \mathcal{R}(t, g)=\mathcal{V}(s, t)
$$

almost everywhere. Considering $\mathcal{R} \in \mathrm{L}\left(\Gamma_{11}, \mathbb{T}\right)$ and $\mathcal{V} \in \mathrm{L}\left(\Gamma_{02}, \mathbb{T}\right)$, this equation can be rewritten as $\mathrm{d}^{\mathrm{h}} \mathcal{R}=\mathrm{d}_{1}^{\mathrm{v}} \mathcal{V}$.

On the other hand, $\Delta(I) \subset M \otimes I$ and

$$
\Delta(Y(s))=\left(\left(\left(\lambda_{s} \otimes 1 \otimes 1\right)\left(\pi_{1} \otimes \iota\right)\left(X_{s}\right)\right) \otimes 1\right)\left(\pi_{1} \otimes \pi_{1}\right) \Delta_{1}(\mathcal{R}(s, \cdot)) .
$$

Hence, we find that

$$
\left(\lambda_{p_{2}(s g)} \otimes 1\right) \pi_{1}(\mathcal{R}(s, g \cdot)) \in I
$$

for almost all $s, g$. But also $Y\left(p_{2}(s g)\right)=\left(\lambda_{p_{2}(s g)} \otimes 1\right) \pi_{1}\left(\mathcal{R}\left(p_{2}(s g), \cdot\right)\right) \in I$. It follows that we can take a measurable function $\mathcal{U}: G_{2} \times G_{1} \rightarrow \mathbb{T}$ such that

$$
\mathcal{R}\left(p_{2}(s g), h\right) \overline{\mathcal{R}}(s, g h) \mathcal{R}(s, g)=\mathcal{U}(s, g)
$$

almost everywhere. Considering $\mathcal{R}, \mathcal{U} \in \mathrm{L}\left(\Gamma_{11}, \mathbb{T}\right)$, this equation can be rewritten as $\mathrm{d}^{\mathrm{v}} \mathcal{R}=\mathrm{d}_{2}^{\mathrm{v}} \mathcal{U}$.

We shift back to additive notation. From the equation $\mathrm{d}^{\mathrm{v}} \mathcal{R}=\mathrm{d}_{2}^{\mathrm{v}} \mathcal{U}$, it follows that $\left(\mathrm{d}_{0}^{\mathrm{v}}-\mathrm{d}_{1}^{\mathrm{v}}+\mathrm{d}_{2}^{\mathrm{v}}-\mathrm{d}_{3}^{\mathrm{v}}\right) \mathrm{d}_{2}^{\mathrm{v}} \mathcal{U}=\mathrm{d}^{\mathrm{v}} \mathrm{d}_{2}^{\mathrm{v}} \mathcal{U}=0$, which yields that $\mathrm{d}_{3}^{\mathrm{v}}\left(\mathrm{d}_{0}^{\mathrm{v}}-\mathrm{d}_{1}^{\mathrm{v}}\right) \mathcal{U}=0$. Hence, $\left(\mathrm{d}_{0}^{\mathrm{v}}-\mathrm{d}_{1}^{\mathrm{v}}\right) \mathcal{U}=0$ and we can take $\mathcal{W} \in \mathrm{L}\left(\Gamma_{01}, \mathbb{T}\right)$ such that $\mathcal{U}=\mathrm{d}_{0}^{\mathrm{v}} \mathcal{W}$. We then find that

$$
\begin{aligned}
\mathrm{d}_{2}^{\mathrm{v}} \mathrm{d}_{0}^{\mathrm{v}} \mathrm{d}^{\mathrm{h}} \mathcal{W} & =\mathrm{d}^{\mathrm{h}} \mathrm{d}_{2}^{\mathrm{v}} \mathcal{U}=\mathrm{d}^{\mathrm{h}} \mathrm{d}^{\mathrm{v}} \mathcal{R}=\mathrm{d}^{\mathrm{v}} \mathrm{d}^{\mathrm{h}} \mathcal{R}=\mathrm{d}^{\mathrm{v}} \mathrm{d}_{1}^{\mathrm{v}} \mathcal{V} \\
& =\left(\mathrm{d}_{0}^{\mathrm{v}}-\mathrm{d}_{1}^{\mathrm{v}}+\mathrm{d}_{2}^{\mathrm{v}}\right) \mathrm{d}_{1}^{\mathrm{v}} \mathcal{V}=\mathrm{d}_{0}^{\mathrm{v}} \mathrm{d}_{1}^{\mathrm{v}} \mathcal{V}=\mathrm{d}_{2}^{\mathrm{v}} \mathrm{d}_{0}^{\mathrm{v}} \mathcal{V}
\end{aligned}
$$

Hence, $\mathcal{V}=\mathrm{d}^{\mathrm{h}} \mathcal{W}$. Define $\widetilde{\mathcal{R}}=\mathcal{R}-\mathrm{d}_{1}^{\mathrm{v}} \mathcal{W}$. Then, $\mathrm{d}^{\mathrm{h}} \widetilde{\mathcal{R}}=0$ as well as $\mathrm{d}^{\mathrm{v}} \widetilde{\mathcal{R}}=0$. So, $\widetilde{\mathcal{R}} \in H^{1}(m . p ., \mathbb{T})$. Moreover, the coideal defined by $\widetilde{\mathcal{R}}$ is generated by the operators

$$
\left(\lambda_{s} \otimes 1\right) \pi_{1}(\widetilde{\mathcal{R}}(s, \cdot))=\overline{\mathcal{W}}(s)\left(\lambda_{s} \otimes 1\right) \pi_{1}(\mathcal{R}(s, \cdot))=\overline{\mathcal{W}}(s) Y(s) \in I .
$$

Writing $Z(s)=\widetilde{\mathcal{R}}\left(\lambda_{s} \otimes 1\right) \widetilde{\mathcal{R}}^{*} \in I$, we get a strongly continuous homomorphism $s \mapsto Z(s)$ satisfying $\theta(Z(s))=\lambda_{s} \otimes Z(s)$. From Landstad's theorem (see 16]), it follows that $I$ is a crossed product von Neumann algebra and, in particular, that $I$ is generated by $I^{\theta}$ and $\left\{Z(s) \mid s \in G_{2}\right\}$. Now, $I^{\theta}=E_{e}=\mathbb{C}$ and we conclude that the coideal defined by $\widetilde{\mathcal{R}}$ is precisely $I$.

Remark 6.4. In the previous proposition we described the full coideals of $(M, \Delta)$ in terms of $H^{1}(m . p ., \mathbb{T})$. There is no hope to describe all coideals of $(M, \Delta)$ using 
cohomological data. This would come down to describe, in the classical setting, all closed subgroups of $G$ in terms of $G_{1}$ and $G_{2}$ if

$$
e \longrightarrow G_{1} \longrightarrow G \longrightarrow G_{2} \longrightarrow e
$$

is an exact sequence. It is clear that we can only describe in such a way a closed subgroup of $G$ whose image in $G_{2}$ is closed.

In the previous proposition, we characterized in a quantum setting the closed subgroups $H$ of $G$ such that the homomorphism from $H$ to $G_{2}$ is in fact a bijective homeomorphism. More generally, it is possible to describe the coideals of the bicrossed product $(M, \Delta)$ whose image in $\mathcal{L}\left(G_{2}\right)$ is, in a certain sense, closed.

\section{Computational methods}

Let $G_{1}, G_{2} \subset G$ be a matched pair of locally compact groups. We want to calculate the group of extensions $H^{2}(m . p ., \mathbb{T})$. Taking into account the Kac exact sequence (Corollary 4.5), we have to calculate $H^{n}(\Gamma, \mathbb{T})$, for $n=2,3$ and $\Gamma=$ $G, G_{1}, G_{2}$. David Wigner developed in [25] the necessary tools.

Let $G$ be a locally compact group. The short exact sequence $0 \rightarrow \mathbb{Z} \rightarrow \mathbb{R} \rightarrow$ $\mathbb{T} \rightarrow 0$ yields the long exact cohomology sequence

$$
\cdots \rightarrow H^{n}(G, \mathbb{Z}) \rightarrow H^{n}(G, \mathbb{R}) \rightarrow H^{n}(G, \mathbb{T}) \rightarrow H^{n+1}(G, \mathbb{Z}) \rightarrow \cdots
$$

So, we have to calculate $H^{n}(G, \mathbb{R})$ and $H^{n}(G, \mathbb{Z})$.

In order to do so, we explain the approach of David Wigner. We introduce a special class of Polish $\mathbb{Z}$-modules having property $F$, which means that for every short exact sequence $0 \rightarrow A \rightarrow B \stackrel{\pi}{\rightarrow} C \rightarrow 0$, $\pi$ has the homotopy lifting property for finite-dimensional paracompact spaces.

We consider $H^{n}(G, A)$ for

- locally compact, $\sigma$-compact groups $G$ of finite dimension (in the sense of Lebesgue covering dimension),

- Polish $G$-modules $A$ with property $F$.

In such a situation, we define on the locally compact space $G^{n}$ the sheaf $\mathcal{A}^{n}$ of continuous functions to $A$, i.e. $\mathcal{A}^{n}(U)=\{f: U \rightarrow A \mid f$ continuous $\}$ for all $U \subset G^{n}$ open. Since the usual face operators $\partial_{i}: G^{n} \rightarrow G^{n-1}, i=0, \ldots, n$, are continuous, we get a bicomplex $\left(C^{k}\left(G^{n}, \mathcal{A}^{n}\right)\right)_{k, n \geq 0}$ :

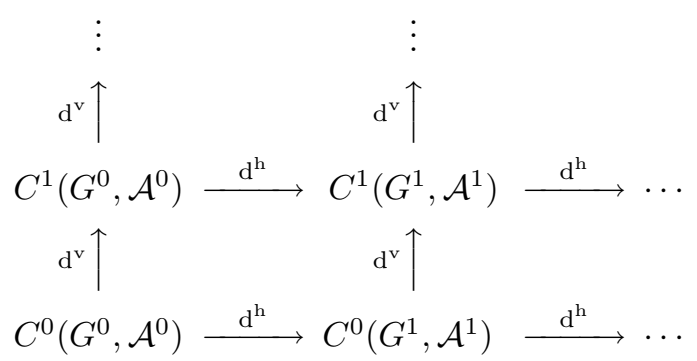

Here, $C^{k}\left(G^{n}, \mathcal{A}^{n}\right)$ denotes the semisimplicial $k$-cochains of sheaf cohomology (see 6], Chapter II, end of Section 2), $\mathrm{d}^{\mathrm{v}}$ is the coboundary of sheaf cohomology and $\mathrm{d}^{\mathrm{h}}$ is the coboundary of group cohomology. Wigner shows that the cohomology of the total bicomplex is precisely $H(G, A)$.

One can write an explicit isomorphism as follows: define $\mathcal{A}_{\text {Borel }}^{n}$ to be the sheaf on $G^{n}$ defined by $\mathcal{A}^{n}(U)=\{f: U \rightarrow A \mid f$ Borel measurable $\}$ for all $U \subset G^{n}$ 
open. We get a bicomplex $\left(C^{k}\left(G^{n}, \mathcal{A}_{\text {Borel }}^{n}\right)\right)$. Using the inclusion $\mathcal{A}^{n} \hookrightarrow \mathcal{A}_{\text {Borel }}^{n}$ we get a cochain transformation $\left(C^{k}\left(G^{n}, \mathcal{A}^{n}\right)\right) \rightarrow\left(C^{k}\left(G^{n}, \mathcal{A}_{\text {Borel }}^{n}\right)\right)$ which is a total cohomology isomorphism. Since the sheafs $\mathcal{A}_{\text {Borel }}^{n}$ are flabby, the total cohomology of the second bicomplex is supported on the first row and gives precisely the Borel cohomology of $G$, defined using the complex $\left(\mathcal{F}_{\text {Borel }}\left(G^{n}, A\right)\right)_{n}$.

Suppose now that $A$ is contractible. Then, the sheafs $\mathcal{A}^{n}$ are flabby and the cohomology of the total bicomplex of (24) is supported on the first row. By definition, we get that $H(G, A)=H_{\text {cont }}(G, A)$. Suppose moreover that $A$ is a finite-dimensional vector space and that $G$ is a Lie group with finitely many connected components. Let $K \subset G$ be a maximal compact subgroup. Using results of Hochschild \& Mostow and Van Est, we get that $H(G, A)=H_{\text {cont }}(G, A)=H(\mathfrak{g}, K, A)$, the Lie algebra cohomology relative to $K$ as defined in [11, Chapitre II, $\mathrm{n}^{\circ} 3.6$.

In our examples, we will look at $A=\mathbb{R}$ and $G$ will be low dimensional, so that $H(\mathfrak{g}, K, A)$ is perfectly computable.

On the opposite suppose that $A$ is a discrete $G$-module. Let $E G \rightarrow B G$ be a principal universal $G$-bundle with paracompact base (see [12]). Let $\mathcal{B}^{n}$ denote the sheaf of continuous (hence, locally constant) functions on $E G \times G^{n}$, i.e. $\mathcal{B}^{n}(U)=$ $\{f: U \rightarrow A \mid f$ continuous $\}$ for all $U \subset E G \times G^{n}$ open. Define the face operators $\partial_{i}^{\mathrm{h}}: E G \times G^{n} \rightarrow E G \times G^{n-1}, i=0, \ldots, n$, by the formula

$$
\partial_{i}^{\mathrm{h}}\left(x, g_{1}, \ldots, g_{n}\right)=\left\{\begin{array}{l}
\left(x \cdot g_{1}, g_{2}, \ldots, g_{n}\right) \quad \text { if } i=0 \\
\left(x, g_{1}, \ldots, g_{i} g_{i+1}, \ldots, g_{n}\right) \text { if } i=1, \ldots, n-1 \\
\left(x, g_{1}, \ldots, g_{n-1}\right) \text { if } i=n
\end{array}\right.
$$

Proceeding as above, we get a bicomplex $\left(C^{k}\left(E G \times G^{n}, \mathcal{B}^{n}\right)\right)_{k, n \geq 0}$. Using the projection $E G \times G^{n} \rightarrow G^{n}$ we get a cochain transformation $\left(C^{k}\left(G^{n}, \mathcal{A}^{n}\right)\right) \rightarrow$ $\left(C^{k}\left(E G \times G^{n}, \mathcal{B}^{n}\right)\right)$. Since $E G$ is contractible and $A$ discrete, the homotopy invariance of sheaf cohomology implies that this cochain transformation induces a cohomology isomorphism in every column. Hence, we get an isomorphism in total cohomology. Next (and this argument is slightly delicate; see page 92 in Wigner's paper [25]), we can use local sections for the map $E G \rightarrow B G$ to show that the total cohomology of the bicomplex $\left(C^{k}\left(E G \times G^{n}, \mathcal{B}^{n}\right)\right)$ is supported on the first column. As a conclusion, we get that $H(G, A)$ is precisely the sheaf cohomology of $B G$ with coefficients in the locally constant sheaf $A$ on $B G$.

As an application, we have the following result.

Proposition 7.1. Let $G_{1}, G_{2} \subset G$ be a matched pair and suppose that $G$ is finite dimensional. Let $E G \rightarrow B G$ be a principal universal $G$-bundle with paracompact base. We get principal universal $G_{i}$-bundles $E G \rightarrow B G_{i}$ and continuous maps $B G_{i} \rightarrow B G$. The Kac cohomology $H(m . p ., A)$ with coefficients in a discrete, trivial $G$-module $A$ is the singular cohomology of the mapping cone of $B G_{1} \sqcup B G_{2} \rightarrow B G$ with coefficients in $A$.

Proof. Denote, on all kinds of spaces, the constant sheaf $A$ by $\mathcal{A}$ and the sheaf of Borel functions to $A$ by $\mathcal{A}_{\text {Borel }}$. Consider the diagram (25) below. The arrows labeled with (1) are defined by (18). The arrows labeled with (2) are the obvious ones. Both are cohomology isomorphisms by the Buchsbaum's criterion (see Preliminaries). The arrows labeled with (3) are cohomology isomorphisms by homotopy invariance of sheaf cohomology, as explained above. Finally, the arrows 


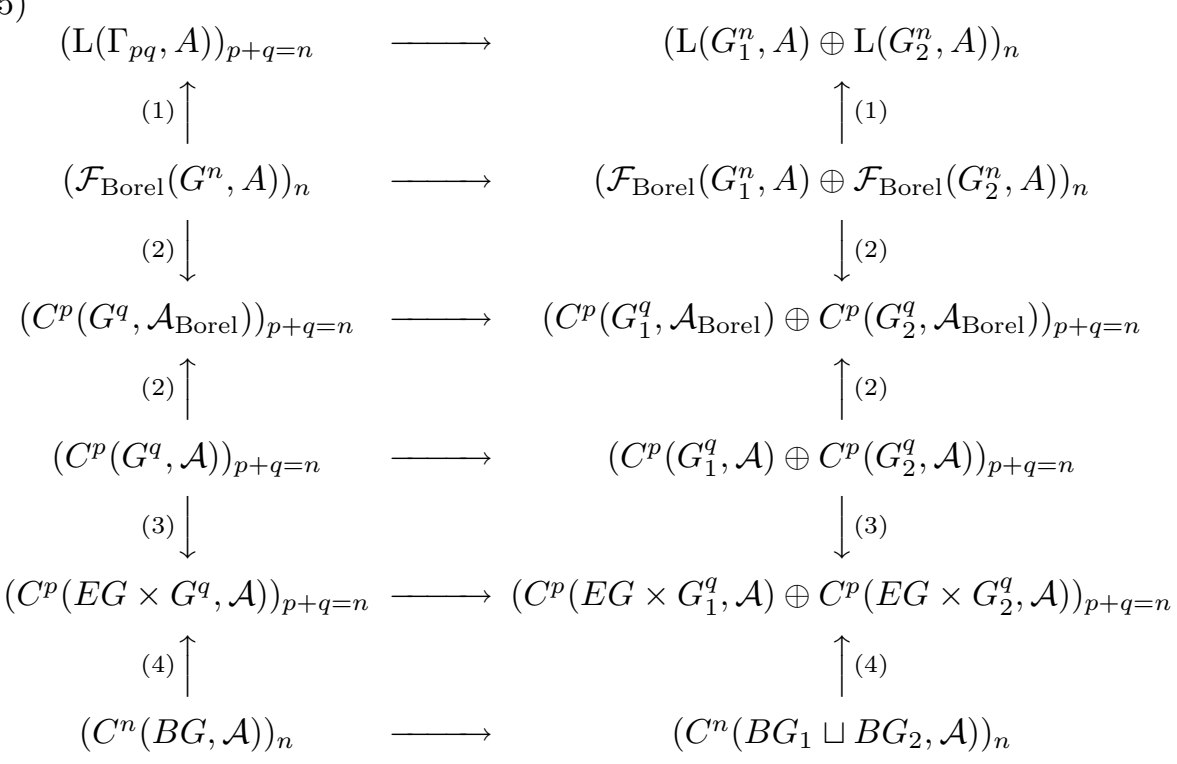

FiguRE 1. Diagram for the proof of Proposition 7.1

labeled with (4) are cohomology isomorphisms by Wigner's argument on page 92 of $[25$.

The cohomology of the mapping cone of the cochain transformation on the first line of the diagram in Figure 1 is by definition the Kac cohomology $H(m . p ., A)$. The cohomology of the mapping cone of the cochain transformation on the last line of the diagram is precisely the singular cohomology of the mapping cone of $B G_{1} \sqcup B G_{2} \rightarrow B G$ with coefficients in $A$. So, both cohomologies are isomorphic.

\section{EXAMPLES}

8.1. Matched pairs of low-dimensional Lie groups. In 24], matched pairs of real Lie algebras $\mathfrak{g}_{1}, \mathfrak{g}_{2} \subset \mathfrak{g}$ have been classified for $\operatorname{dim} \mathfrak{g}_{1} \leq 2$ and $\mathfrak{g}_{2}=\mathbb{R}$. All these matched pairs have exponentiations to matched pairs of Lie groups, in the sense of Definition 2.1. The exponentiations that are as connected as possible (but not always connected) have been given explicitly in 24. The group of extensions was calculated on the Lie algebra level and explicit exponentiations to cocycles satisfying (5) have been given. Nevertheless, there were not the necessary tools at hand to prove that these cocycles really represented exactly the elements of the group of extensions in the sense of Definition 3.1 .

Using the methods developed above, we will correctly compute the groups of extensions for the matched pairs of [24], Theorem 5.1.

Remark that if $G_{1}, G_{2}$ and $G$ are connected and have no non-trivial compact subgroups, all cohomologies with coefficients in $\mathbb{Z}$ vanish and those with coefficients in $\mathbb{R}$ reduce to Lie algebra cohomology. So, the group of extensions $H^{2}(m . p ., \mathbb{T})$ is isomorphic to the group of Lie bialgebra extensions for the matched pair $\mathfrak{g}_{1}, \mathfrak{g}_{2} \subset \mathfrak{g}$, as defined in [20]. In particular, the group of extensions for the cases $1-3$ of [24], Theorem 5.1, are either $\mathbb{R}$ or 0 , as described in [24], Proposition 6.2. 
Next, we have to look at case 4 of [24], Theorem 5.1. A first class of matched pairs depends on a parameter $d \notin\{0,1\}$. We have

$$
\begin{aligned}
G & =\left\{\left(\begin{array}{ccc}
s & 0 & x \\
0 & s^{d} & y \\
0 & 0 & 1
\end{array}\right) \mid s \neq 0, x, y \in \mathbb{R}\right\} \quad \text { where } \quad s^{d}=\operatorname{Sgn}(s)|s|^{d}, \\
G_{1} & =\left\{\left(\begin{array}{ccc}
s & 0 & 0 \\
0 & s^{d} & y \\
0 & 0 & 1
\end{array}\right) \mid s \neq 0, y \in \mathbb{R}\right\}, \quad G_{2}=\left\{\left(\begin{array}{ccc}
s & 0 & s-1 \\
0 & s^{d} & 0 \\
0 & 0 & 1
\end{array}\right) \mid s \neq 0\right\} .
\end{aligned}
$$

Using the results of Section 7 , we get that $H^{2}\left(G_{1}, \mathbb{T}\right)=H^{2}\left(G_{2}, \mathbb{T}\right)=0$, that $H^{3}\left(G_{1}, \mathbb{R}\right)=H^{3}\left(G_{2}, \mathbb{R}\right)=0$ and that the arrow $H^{4}(G, \mathbb{Z}) \rightarrow H^{4}\left(G_{1}, \mathbb{Z}\right)$ is an isomorphism. Further, we have

$$
H^{3}(G, \mathbb{R})= \begin{cases}\mathbb{R} & \text { if } d=-1 \\ 0 & \text { otherwise }\end{cases}
$$

All this information, together with the Kac exact sequence, shows that the group of extensions is $\mathbb{R}$ for $d=-1$ and 0 otherwise. An immediate verification shows that, for the case $d=-1$, the cocycles are exactly given by Proposition 6.3 in [24].

A second class of matched pairs depends on a parameter $b \in \mathbb{R}$. We have

$$
\begin{aligned}
G & =\left\{\left(\begin{array}{ccc}
s & b s \log |s| & x \\
0 & s & y \\
0 & 0 & 1
\end{array}\right) \mid s \neq 0, x, y \in \mathbb{R}\right\}, \\
G_{1} & =\left\{\left(\begin{array}{ccc}
s & b s \log |s| & x \\
0 & s & 0 \\
0 & 0 & 1
\end{array}\right) \mid s \neq 0, x \in \mathbb{R}\right\}, \\
G_{2} & =\left\{\left(\begin{array}{ccc}
s & b s \log |s| & 0 \\
0 & s & s-1 \\
0 & 0 & 1
\end{array}\right) \mid s \neq 0\right\} .
\end{aligned}
$$

With exactly the same reasoning as above, we find that the group of extensions is trivial, since now $H^{3}(G, \mathbb{R})=0$.

Finally, consider three more interesting cases. Before writing the corresponding Lie groups $G_{1}, G_{2} \subset G$, we compute and write generators for $H^{4}(\mathbb{T}, \mathbb{Z})$ and $H^{4}(\mathbb{Z} / 2 \mathbb{Z} \ltimes \mathbb{T}, \mathbb{Z})$, where $\mathbb{Z} / 2 \mathbb{Z}$ acts by taking the inverse. It is well known that $B \mathbb{T}=P \mathbb{C}^{\infty}$, the infinite-dimensional projective plane. Under the cup product $H\left(P \mathbb{C}^{\infty}, \mathbb{Z}\right)=\mathbb{Z}[X]$, the polynomial ring over $\mathbb{Z}$. In particular, if $x$ is a generator for $H^{2}\left(P \mathbb{C}^{\infty}, \mathbb{Z}\right), x \cup x$ will be a generator for $H^{4}\left(P \mathbb{C}^{\infty}, \mathbb{Z}\right)$. Also the measurable cohomology $H(G, \mathbb{Z})$ has a cup product: if $\alpha \in \mathrm{L}\left(G^{n}, \mathbb{Z}\right)$ and $\beta \in \mathrm{L}\left(G^{m}, \mathbb{Z}\right)$ are cocycles, we define

$$
(\alpha \cup \beta)\left(g_{1}, \ldots, g_{n+m}\right)=\alpha\left(g_{1}, \ldots, g_{n}\right) \beta\left(g_{n+1}, \ldots, g_{n+m}\right) .
$$

One can verify that the isomorphism $H(G, \mathbb{Z}) \cong H(B G, \mathbb{Z})$ preserves cup products. We conclude that, if $\omega$ is a generator for $H^{2}(G, \mathbb{Z}) \cong \mathbb{Z}$, then $\omega \cup \omega$ is a generator for $H^{4}(G, \mathbb{Z})$. But, a generator $\omega$ for $H^{2}(\mathbb{T}, \mathbb{Z})$ is well known, since it comes from the extension $0 \rightarrow \mathbb{Z} \rightarrow \mathbb{R} \rightarrow \mathbb{T} \rightarrow 0$. We make the following choice that will be 
useful later:

for $-\pi \leq s, t<\pi$, we define $\omega(\exp (i t), \exp (i s))=\left\{\begin{array}{l}1 \text { if } s+t \geq \pi, \\ 0 \text { if }-\pi \leq s+t<\pi, \\ -1 \quad \text { if } s+t<-\pi\end{array}\right.$

An explicit generator for $H^{4}(\mathbb{T}, \mathbb{Z})$ is then given by

$$
\alpha\left(\lambda_{1}, \lambda_{2}, \lambda_{3}, \lambda_{4}\right)=\omega\left(\lambda_{1}, \lambda_{2}\right) \omega\left(\lambda_{3}, \lambda_{4}\right) .
$$

We turn next to $\mathbb{Z} / 2 \mathbb{Z} \ltimes \mathbb{T}$. Consider more generally a semi-direct product $G:=$ $\Gamma \ltimes K$, with $\Gamma$ discrete. Using the subgroups $\Gamma$ and $K$, we have a matched pair and hence, we have the bicomplex (17) to compute the measurable cohomology $H(G, A)$. Associated with this bicomplex is a spectral sequence (the Lyndon-HochschildSerre spectral sequence), which makes perfect sense because $\Gamma$ is discrete. We have $E_{p, q}^{2}=H^{p}\left(\Gamma, H^{q}(K, A)\right)$, where we consider $H^{q}(K, A)$ as a discrete $\Gamma$-module. In our concrete case, with $\Gamma=\mathbb{Z} / 2 \mathbb{Z}$ and $K=\mathbb{T}$, we find $E_{2}^{4,0}=\mathbb{Z} / 2 \mathbb{Z}, E_{2}^{3,1}=E_{2}^{2,2}=$ $E_{2}^{1,3}=0$ and $E_{2}^{0,4}=\mathbb{Z}$. Since the action of $\mathbb{Z} / 2 \mathbb{Z}$ is trivial on the element $\alpha \in$ $\mathrm{L}\left(\mathbb{T}^{4}, \mathbb{Z}\right)$, we can conclude that $H^{4}(\mathbb{Z} / 2 \mathbb{Z} \ltimes \mathbb{T}, \mathbb{Z}) \cong \mathbb{Z} / 2 \mathbb{Z} \oplus \mathbb{Z}$ and more specifically, the restriction homomorphism $H^{4}(\mathbb{Z} / 2 \mathbb{Z} \ltimes \mathbb{T}, \mathbb{Z}) \rightarrow H^{4}(\mathbb{Z} / 2 \mathbb{Z}, \mathbb{Z}) \oplus H^{4}(\mathbb{T}, \mathbb{Z})$ is an isomorphism.

Consider the following matched pair:

$$
\begin{aligned}
G & =\left\{X \in M_{2}(\mathbb{R}) \mid \operatorname{det} X= \pm 1\right\} \quad \bmod \{ \pm 1\}, \\
G_{1} & =\left\{\left(\begin{array}{cc}
|a| & 0 \\
x & \frac{1}{a}
\end{array}\right) \mid a \neq 0, x \in \mathbb{R}\right\} \quad \bmod \{ \pm 1\}, \\
G_{2} & =\left\{\left(\begin{array}{cc}
|s| & \frac{1}{2}\left(|s|-\frac{1}{s}\right) \\
0 & \frac{1}{s}
\end{array}\right) \mid s \neq 0\right\} \quad \bmod \{ \pm 1\} .
\end{aligned}
$$

We get that $H^{3}(G, \mathbb{R})=0$ and $H^{2}\left(G_{1}, \mathbb{T}\right)=H^{2}\left(G_{2}, \mathbb{T}\right)=0$. We can then easily conclude from the Kac exact sequence that the sequence $0 \rightarrow H^{2}($ m.p., $\mathbb{T}) \rightarrow$ $H^{4}(G, \mathbb{Z}) \rightarrow H^{4}\left(G_{1}, \mathbb{Z}\right) \oplus H^{4}\left(G_{2}, \mathbb{Z}\right)$ is exact. Consider in the obvious way $\mathbb{Z} / 2 \mathbb{Z} \subset$ $G_{1}$ and $\mathbb{Z} / 2 \mathbb{Z} \subset G_{2}$. Both embeddings of $\mathbb{Z} / 2 \mathbb{Z}$ in $G$ are conjugate in $G$. Since conjugation by an element of $G$ acts trivially on $H^{4}(G, \mathbb{Z})$, we conclude that the sequence $0 \rightarrow H^{2}(m . p ., \mathbb{T}) \rightarrow H^{4}(G, \mathbb{Z}) \rightarrow H^{4}\left(G_{1}, \mathbb{Z}\right)$ is exact. Using the maximal compact subgroup $\mathbb{Z} / 2 \mathbb{Z} \ltimes \mathbb{T} \subset G$, it follows from this that $0 \rightarrow H^{2}(m . p ., \mathbb{T}) \rightarrow$ $H^{4}(\mathbb{Z} / 2 \mathbb{Z} \ltimes \mathbb{T}, \mathbb{Z}) \rightarrow H^{4}(\mathbb{Z} / 2 \mathbb{Z}, \mathbb{Z})$ is exact. From the remarks above, we conclude that $H^{2}(m . p ., \mathbb{T}) \rightarrow H^{4}(\mathbb{T}, \mathbb{Z})$ is an isomorphism. Hence, the group of extensions is $H^{2}(m . p ., \mathbb{T})=\mathbb{Z}$. In [24], Proposition 6.3, explicit pairs of 2-cocycles $\left(\mathcal{U}_{n}, 1\right)_{n \in \mathbb{Z}}$ have been constructed for the matched pair $G_{1}, G_{2} \subset G$. Using Remark 4.2 and the final formula on page 171 as well as the first formula on page 172 of [24, we can check that the image of $\left(\mathcal{U}_{n}, 1\right)$ under the isomorphism $H^{2}(m . p ., \mathbb{T}) \rightarrow H^{4}(\mathbb{T}, \mathbb{Z})$ is precisely $-4 n \alpha$, where $\alpha$ is defined by (26). Once one is able to perform such a calculation, one can see as well how to change the function $f$ in 24] in order to get exactly the whole of $H^{2}(m . p ., \mathbb{T})$.

Next, consider the matched pair with

$$
\begin{aligned}
G & =\mathrm{PSL}_{2}(\mathbb{R}), \quad G_{1}=\left\{\left(\begin{array}{cc}
a & x \\
0 & \frac{1}{a}
\end{array}\right) \mid a>0, x \in \mathbb{R}\right\} \quad \bmod \{ \pm 1\}, \\
G_{2} & =\left\{\left(\begin{array}{ll}
1 & 0 \\
s & 1
\end{array}\right) \mid s \in \mathbb{R}\right\} \quad \bmod \{ \pm 1\} .
\end{aligned}
$$


Considering the maximal compact subgroup $\mathbb{T} \subset G$, we immediately get that $H^{2}(m . p ., \mathbb{T}) \rightarrow H^{4}(\mathbb{T}, \mathbb{Z})$ is an isomorphism. The explicit cocycles $\left(\mathcal{U}_{n}, 1\right)$ found in [24] are mapped to $2 n \alpha$ under this isomorphism. Again, it is not hard to find explicit formulas for cocycles giving exactly the whole of $H^{2}(m . p ., \mathbb{T})$.

Finally, we look at the matched pair

$$
\begin{aligned}
G=\mathrm{PSL}_{2}(\mathbb{R}), \quad G_{1} & =\left\{\left(\begin{array}{cc}
a & x \\
0 & \frac{1}{a}
\end{array}\right) \mid a>0, x \in \mathbb{R}\right\} \quad \bmod \{ \pm 1\}, \\
G_{2} & =\left\{\left(\begin{array}{cc}
\cos t & \sin t \\
-\sin t & \cos t
\end{array}\right) \mid t \in \mathbb{R}\right\} \quad \bmod \{ \pm 1\} .
\end{aligned}
$$

From the Kac exact sequence, it follows that the sequence $0 \rightarrow H^{2}(m . p ., \mathbb{T}) \rightarrow$ $H^{4}(G, \mathbb{Z}) \rightarrow H^{4}\left(G_{2}, \mathbb{Z}\right)$ is exact. Since $H^{4}(G, \mathbb{Z}) \rightarrow H^{4}\left(G_{2}, \mathbb{Z}\right)$ is an isomorphism, we get $H^{2}(m . p ., \mathbb{T})=0$. This explains why it is impossible in [24] to exponentiate the cocycles from the Lie algebra to the Lie group level.

In the final matched pair above, we can take the associated matched pair of Lie algebras $\mathfrak{g}_{1}, \mathfrak{g}_{2} \subset \mathfrak{g}$. If we take $\widetilde{G}$ to be the connected, simply connected Lie group with Lie algebra $\mathfrak{g}$ and $\widetilde{G}_{1}, \widetilde{G}_{2}$ to be the connected Lie subgroups with Lie algebras $\mathfrak{g}_{1}, \mathfrak{g}_{2}$, we get a matched pair such that $\widetilde{G}, \widetilde{G}_{1}, \widetilde{G}_{2}$ are connected and without compact subgroups. So, we conclude as above that $H^{2}(m . p ., \mathbb{T})$ is isomorphic to the group of Lie bialgebra extensions, i.e. $\mathbb{R}$ as stated in Proposition 6.2 of [24].

8.2. Some other examples. Let $G$ be a semi-simple Lie group with finite center, such that in its Iwasawa decomposition $G=K A N, K$ is a maximal compact subgroup. Writing $G_{1}=K$ and $G_{2}=A N$, we get a matched pair of Lie groups. Since $G_{2}$ is contractible, we get that $H^{n}\left(G_{2}, \mathbb{Z}\right)=0$ and $H^{n}(G, \mathbb{Z}) \rightarrow H^{n}\left(G_{1}, \mathbb{Z}\right)$ is an isomorphism for all $n \geq 1$. It follows from the Kac exact sequence that $H^{n}(m . p ., \mathbb{Z})=0$ for $n \geq 1$. Hence, $H^{n}(m . p ., \mathbb{R}) \rightarrow H^{n}(m . p ., \mathbb{T})$ is an isomorphism. But,

$$
\begin{aligned}
H^{2}(G, \mathbb{R}) & \rightarrow H^{2}\left(G_{1}, \mathbb{R}\right) \oplus H^{2}\left(G_{2}, \mathbb{R}\right) \rightarrow H^{2}(m . p ., \mathbb{R}) \\
& \rightarrow H^{3}(G, \mathbb{R}) \rightarrow H^{3}\left(G_{1}, \mathbb{R}\right) \oplus H^{3}\left(G_{2}, \mathbb{R}\right)
\end{aligned}
$$

is an exact sequence of vector spaces. Moreover, $H^{n}\left(G_{1}, \mathbb{R}\right)=0$ for $n \geq 1$. Writing $\mathfrak{s}=\mathfrak{a} \oplus \mathfrak{n}$ for the Lie algebra of $G_{2}$ and $\mathfrak{k}$ for the Lie algebra of $K$, it follows that the group of extensions is isomorphic with

$$
\begin{aligned}
& H^{2}(m . p ., \mathbb{T}) \cong \operatorname{Coker}\left(H^{2}(\mathfrak{g}, \mathfrak{k}, \mathbb{R}) \rightarrow H^{2}(\mathfrak{s}, \mathbb{R})\right) \\
& \oplus \operatorname{Ker}\left(H^{3}(\mathfrak{g}, \mathfrak{k}, \mathbb{R}) \rightarrow H^{3}(\mathfrak{s}, \mathbb{R})\right) .
\end{aligned}
$$

If we take $G=\mathrm{SL}_{2}(\mathbb{R})$, we immediately get that the group of extensions is trivial. For $G=\mathrm{SL}_{2}(\mathbb{C})$, a direct calculation gives $H^{2}(\mathfrak{s}, \mathbb{R})=H^{3}(\mathfrak{s}, \mathbb{R})=0$, while $H^{3}(\mathfrak{g}, \mathfrak{k}, \mathbb{R})=\mathbb{R}$. So, in this case $H^{2}(m . p ., \mathbb{T})=\mathbb{R}$. Observe that we deal precisely with the matched pair considered by Majid in [18].

In [5], a class of matched pairs is constructed as follows: let $\mathcal{A}$ be a locally compact ring such that $\mathcal{A} \backslash \mathcal{A}^{\star}$ has (additive) Haar measure zero, where $\mathcal{A}^{\star}$ is the group of units. Let $G$ be the group of affine transformations of $\mathcal{A}$, i.e. the locally compact group with underlying space $\mathcal{A}^{\star} \times \mathcal{A}$ and product $(a, x) \cdot(b, y)=(a b, x+a y)$. 
The subgroups $G_{1}, G_{2}$ will be the subgroups of transformations fixing 0 and -1 , respectively. This means that $G_{1}$ and $G_{2}$ consist of the elements $(a, 0)$ and $(b, b-1)$ for $a, b \in \mathcal{A}^{\star}$.

If we take $\mathcal{A}=\mathbb{R}$, we easily get that $H^{2}(m . p ., \mathbb{T})=0$. If $\mathcal{A}=\mathbb{C}$, we observe that in the commutative diagram

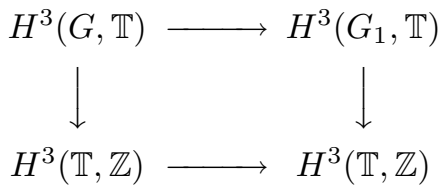

the vertical arrows and the lower horizontal arrow are isomorphisms. Since $H^{2}\left(G_{1}, \mathbb{T}\right)$ $=H^{2}\left(G_{2}, \mathbb{T}\right)=0$, we can conclude that $H^{2}(m \cdot p ., \mathbb{T})=0$.

\section{REFERENCES}

[1] N. Andruskiewitsch, Notes on extensions of Hopf algebras. Can. J. Math. 48 (1996), 3-42. MF 1382474 (97c:16046)

[2] W. Arveson, An invitation to $\mathrm{C}^{*}$-algebras. Graduate Texts in Mathematics, No. 39. Springer-Verlag, New York-Heidelberg, 1976. MR.0512360 (58:23621)

[3] S. BaAj \& G. Skandalis, Unitaires multiplicatifs et dualité pour les produits croisés de C*-algèbres. Ann. Scient. Ec. Norm. Sup., 4e série, 26 (1993), 425-488. MR1235438 (94e:46127)

[4] S. BaAj \& G. Skandalis, Transformations pentagonales. C.R. Acad. Sci., Paris, Sér. I 327 (1998), 623-628. MR 1652717 (99k:28018)

[5] S. BaAJ, G. Skandalis \& S. Vaes, Non-semi-regular quantum groups coming from number theory. Commun. Math. Phys. 235 (2003), 139-167. MR1969723 (2004g:46083)

[6] G.E. Bredon, Sheaf theory. McGraw-Hill, New York, 1967. MR0221500 (36:4552)

[7] R. Brown \& K.C. Mackenzie, Determination of a double Lie groupoid by its core diagram. J. Pure Appl. Algebra 80 (1992), 237-272. MR1170713 (93g:55022)

[8] R. Brown \& C.B. Spencer, Double groupoids and crossed modules. Cahiers Topologie Géom. Différentielle 17 (1976), 343-362. MR0440553 (55:13427)

[9] D.A. Buchsbaum, Satellites and universal functors. Ann. of Math. 71 (1960), 199-209. MF 0112905 (22:3751)

[10] J. Dixmier, Dual et quasi-dual d'une algèbre de Banach involutive. Trans. Amer. Math. Soc. 104 (1963), 273-283. MR0139960 (25:3384)

[11] A. Guichardet, Cohomologie des groupes topologiques et des algèbres de Lie. Editions Cedic/Fernand Nathan, Paris, 1980. MR0644979 (83f:22004)

[12] D. Husemoller, Fibre bundles. McGraw-Hill Book Co., New York-London-Sydney, 1966. MR 0229247 (37:4821)

[13] G.I. KAC, Extensions of groups to ring groups. Math. USSR Sbornik 5 (1968), 451-474.

[14] J. Kustermans \& S. Vaes, Locally compact quantum groups. Ann. Scient. Ec. Norm. Sup. 33 (2000), 837-934. MR,1832993 (2002f:46108)

[15] J. Kustermans \& S. VAES, Locally compact quantum groups in the von Neumann algebraic setting. Math. Scand. 92 (1) (2003), 68-92. MR.1951446|(2003k:46081)

[16] M.B. Landstad, Duality theory for covariant systems. Trans. Amer. Math. Soc. 248 (1979), 223-267. MR0522262 (80j:46107)

[17] S. Mac Lane, Homology. Springer-Verlag, Berlin-Göttingen-Heidelberg, 1963. MR0156879 $(28: 122)$

[18] S. MAJID, Physics for algebraists: Non-commutative and non-cocommutative Hopf algebras by a bicrossproduct construction. J. Algebra 130 (1990), 17-64. MR.1045735 (91j:16050)

[19] S. MAJID, Hopf-von Neumann algebra bicrossproducts, Kac algebra bicrossproducts, and the classical Yang-Baxter equations. J. Funct. Anal. 95 (1991), 291-319. MR 1092128 (92b:46088)

[20] A. Masuoka, Extensions of Hopf Algebras and Lie Bialgebras. Trans. of the AMS 352, No. 8 (2000), 3837-3879. MR 1624190 (2000m:17017) 
[21] C.C. Moore, Group extensions and cohomology for locally compact groups. III \& IV. Trans. Amer. Math. Soc. 221 (1976), 1-33 \& 35-58. MR0414775 (54:2867) MR0414776 (54:2868)

[22] P. Schauenburg, Hopf bimodules, coquasibialgebras, and an exact sequence of Kac. Adv. Math. 165 (2002), 194-263. MR.1887584(2003e:16052)

[23] S. VAes \& L. Vainerman, Extensions of locally compact quantum groups and the bicrossed product construction. Adv. in Math. 175 (1) (2003), 1-101. MR1970242 (2004i:46103)

[24] S. VAes \& L. VAinerman, On low-dimensional locally compact quantum groups. In Locally Compact Quantum Groups and Groupoids. Proceedings of the Meeting of Theoretical Physicists and Mathematicians, Strasbourg, February 21 - 23, 2002., Ed. L. Vainerman, IRMA Lectures on Mathematics and Mathematical Physics, Walter de Gruyter, Berlin, New York (2003), pp. 127-187. MR,1976945 (2004f:17024)

[25] D. Wigner, Algebraic cohomology of topological groups. Trans. Amer. Math. Soc. 178 (1973), 83-93. MR0338132(49:2898)

Laboratoire de Mathématiques Pures, Université Blaise Pascal, Bâtiment de Mathématiques, F-63177 Aubière Cedex, France

E-mail address: Saad.Baaj@math.univ-bpclermont.fr

Algèbres d'Opérateurs et Représentations, Institut de Mathématiques de Jussieu, 175, RUe DU Chevaleret, F-75013 Paris, France

E-mail address: skandal@math.jussieu.fr

Algèbres d'Opérateurs et Représentations, Institut de Mathématiques de Jussieu, 175, Rue du Chevaleret, F-75013 Paris, France - and - Department of Mathematics, Katholieke Universiteit Leuven, Celestijnenlaan 200B, B-3001 Leuven, Belgium

E-mail address: vaes@math.jussieu.fr 